\title{
A Geometric Prediction Model of Surface Morphology in Micro-EDM Considering Stochastic Characteristics of Discharge Crater Size
}

\section{Shaojie Hou}

School of Mechatronics Engineering, Harbin Institute of Technology, Harbin

J C Bai ( $\sim$ jichengbai@hit.edu.cn )

Harbin Institute of Technology

\section{Research Article}

Keywords: Micro-EDM, Surface morphology, Geometric simulation, Stochastic characteristic, Discharge crater size

Posted Date: April 8th, 2021

DOI: https://doi.org/10.21203/rs.3.rs-380586/v1

License: (c) (i) This work is licensed under a Creative Commons Attribution 4.0 International License. Read Full License

Version of Record: A version of this preprint was published at The International Journal of Advanced Manufacturing Technology on August 5th, 2021. See the published version at https://doi.org/10.1007/s00170-021-07795-9. 


\title{
A geometric prediction model of surface morphology in micro-EDM considering stochastic characteristics of discharge crater size
}

\author{
Shaojie Hou ${ }^{1}$, Jicheng Bai ${ }^{2 *}$
}

* Corresponding author.

E-mail address: jichengbai@hit.edu.cn tel: +86-18103661699 (J. Bai).

1 School of Mechatronics Engineering, Harbin Institute of Technology, Harbin 150001, Heilongjiang Province, People's Republic of China, E-mail: houshaojie1234@163.com

2* School of Mechatronics Engineering, Harbin Institute of Technology, Harbin 150001, Heilongjiang Province, People's Republic of China, E-mail: jichengbai@hit.edu.cn

\section{Declarations}

\begin{abstract}
The surface topography of micro-EDM is closely related to its application range, so it is important to accurately predict the surface morphology. Most of the existing models do not consider the stochastic characteristics and uncertainty of the discharge process, which could produce great differences with the experimental results. Aiming at these problems, the stochastic characteristic of the discharge crater size is studied, and a simulation model is established to predict the surface topography of EDM considering the stochastic characteristics of crater size. Firstly, the stochastic characteristics of the crater size were investigated through the finite-successive pulses discharge method and characterized from the perspective of probability theory. The stochastic characteristics was explained by analyzing discharge waveforms. Secondly, the response surface method (RSM) was used to map the corresponding relationship between the input machining parameters and the distribution characteristics of crater size. It was observed that the RSM model can accurately predict the distribution characteristics of crater size in the range of parameters selected in this study. Finally, models based on stochastic distribution of crater size (MSDS) and fixed crater size (MFS) were established respectively. By analyzing the characteristics of the simulated surfaces and the machined surface, it was found that the surface features of MSDS are closer to the real surface features. Compared with MFS, the roughness prediction error and the average of overall error of MSDS are reduced by $1.01 \%$ and $18.97 \%$ respectively. The results of this work are helpful to understand the randomness and realize the controllability of EDM.
\end{abstract}

Keywords: Micro-EDM; Surface morphology; Geometric simulation; Stochastic characteristic; Discharge crater size

\section{Introduction}

Micro-electrical discharge machining (micro-EDM) is a kind of machining technology which uses electro-thermal erosion to remove materials [1]. Each pulse discharge will produce a micro crater on the surface of the workpiece, and many craters overlap to form a complex geometry microstructure. Micro-EDM can process almost all conductive materials, which is not affected by the hardness of materials, and it has good machining ability for difficult-to-cut 
materials, for instance, titanium alloy and cemented carbide [2]. Due to these special advantages, micro-EDM is widely used in aerospace and medical fields of film cooling holes and high-quality surface, etc [3-5]. Different from the traditional machining method, the micro-EDM surface is consisted of discharge craters without directional features, hence it's an isotropic surface. The special textured surface formed by discharge craters has some special properties, such as superhydrophobicity, enhanced Raman scattering and so on. The functionality of EDM surface is closely related to its surface morphology, such as surface roughness, surface peak intervals and so on. At present, the functional application of EDM surface is more and more widely extensive, so it is particularly important to realize the accurate prediction of surface morphology.

The micro-EDM process is composed of a series of single spark discharges, hence many simulation models of EDM pay attention to single-spark discharge model to predicate surface roughness. Kiran and Joshi [6] developed a single discharge erosion model to predict the configuration of craters, using the energy distribution between electrode and workpiece, and they used an earlier surface roughness model to predict the surface roughness of workpiece. Salonitis et al. [7] developed a thermal based model to predicate the material removal rate and surface roughness, and deduced the average surface roughness when two or more discharge craters overlapped based on the single discharge crater obtained through the model. Zhang et al. [8] observed through microscope that the discharge crater depth is about 3 times that of the average surface roughness, and their roughness model based on individual craters is in good agreement with the actual surface roughness to a certain extent. However, the EDM surface is the result of a superposition of discharge craters, and therefore the multi-spark discharge model has more advantages in surface morphology prediction. Tan and Yeo [9] used a symmetric distribution of discharge craters to estimate the upper and lower bounds of $\mathrm{R}_{\max }$, and found that the prediction of the model is more accurate in the case of larger discharge energy. Kurnia et al. [10] proposed a geometric model to estimate the surface roughness based on the uniformly distributed craters, with the average error of $R_{a}$ and $R_{t}$ being $6.5 \%$ and $4.5 \%$ separately compared with the experimental results. However, the assumptions of symmetrical distribution and uniform distribution of discharge craters do not reflect the stochastic nature for location of EDM, hence the application scope of the models is small. In EDM, the distribution of location of discharge craters is of great uncertainty, as it must be considered in order to improve the prediction accuracy of the simulation model. Wang et al. [11] developed a multi-spark model to study the machining process of ultrasonic vibration-magnetic field assisted WEDM, the discharge located at the point where the gap width is shortest between the wire electrode and workpiece, and the predicted surface roughness is in good agreement with the actual surface roughness of the workpiece. Zhao et al. [12] developed a geometric model for the EDM die-sinking process, and the average volume of the discharge crater is applied to this 
model. They used several kinds of discharge craters with different morphologies, and found that the surface roughness of the model was greatly affected by the shape characteristics of discharge craters. Izquierdo et al. [13] have taken the random distribution of discharge positions into account, and the adjusted simulation results show that the errors are below 10\% compared with the measured results. Liu and Guo [14] developed a muti-spark discharge model for die-sinking EDM considering the discharge positions are randomly distributed, to simulate the evolutionary process of EDM surface morphology. However, it is not enough to consider only the stochastic nature of discharge positions, in another work, Jithin et al. $[15,16]$ assumed that the multiple sparks are randomly generated with respect to discharge location and discharge energy, and the distribution of surface roughness shows agreement with that of experimental results under low pulse width, while the error increases under larger pulse width. However, the crater size has great randomness, and the existing models still have some gaps for improvement due to the lack of the assumptions of crater size which is closer to the real situation. Furthermore, the models only focus on the morphology characteristics perpendicular to the surface (such as roughness), but pay little attention to the morphology characteristics parallel to the surface.

The multi-spark simulation model and machining process are based on the overlap of the single discharge crater, hence scholars have done a lot of research on the discharge craters. Li et al. found that the discharge crater became smaller as the gap width increases in the case of the same discharge energy [17], which clarified the effect of the influence of the gap width on the corrosion depth and depth error of the workpiece material in EDM milling [18], they also found that the discharge process is tend to be interrupted as the gap width increases. Mohammadreza et al. [19] compared the crater sizes obtained by thermal simulations and experiments to analyze the influence of the machining parameters on the flushing efficiency of plasma tunnel; they found that flushing efficiency of plasma tunnel increases with the increase of discharge current and decreases with the increase of pulse width. Perveen and Jahan [20] used the Box Behnken design method to predict the crater size with an RC power supply and Ti alloy as the workpiece, and they established a regression equation to predict the crater size. Paul et al. [21] studied the influence of process variables on crater size in dry-EDM, and found that the capacitance of RC circuit and pulse frequency are significant factors with the ANOVA method. Carlos and Patricio [22] found that the crater size increases with the increase of discharge current, and they take the diameter of the crater as a good index to characterize the surface morphology of parts in die-sinking EDM. Liu et al. [23] investigated the effect of pulse width on the discharge crater diameter. It can be concluded from the above literature that the gap width, pulse width and current have a significant impact on the size of the discharge crater, so the experiment will be designed with these three parameters as factors in this paper; furthermore, the average value of discharge crater size is taken as the statistical result, but this statistical method cannot reflect the stochastic characteristics 
of EDM process, because even under the same machining parameters, the crater size also has great differences [22,24,25]. Therefore, a more effective statistical method is needed to describe the size discrepancy of discharge craters.

In this paper, firstly, the stochastic distribution characteristics of crater size are calculated and described by the probability density function. Secondly, the influence of peak current, pulse width and gap width on crater size distribution is studied by response surface design (RSM), and the regression equation for predicting crater size is established. Finally, the geometric model of EDM surface topography is established, and the crater size described by probability density function is applied to the model. The effectiveness of the proposed simulation model is verified by comparing with ordinary simulation model and the experimental results.

\section{Experimental details}

\subsection{Experimental equipment}

The experiments were conducted with a self-made micro-EDM machine tool as shown in Fig. 1a. The resolution of the machine tool is $0.1 \mu \mathrm{m}$ in $\mathrm{X}, \mathrm{Y}$ and $\mathrm{Z}$ directions. The maximum rotational velocity of the spindle is $30000 \mathrm{rpm}$, of which the radial runout is less than $1 \mu \mathrm{m}$. The tool electrode manufacturing module and vision inspection module based on the CCD camera are integrated into the machine tool, enabling the tool electrode to be fabricated and measured easily. In the experiment, a self-made impulse generator controlled by one-chip computer was used, the power supply could generate any number of pulses, the circuit diagram for the experiment was illustrated in Fig. 1b, and the finite-successive pulses discharge method was used.
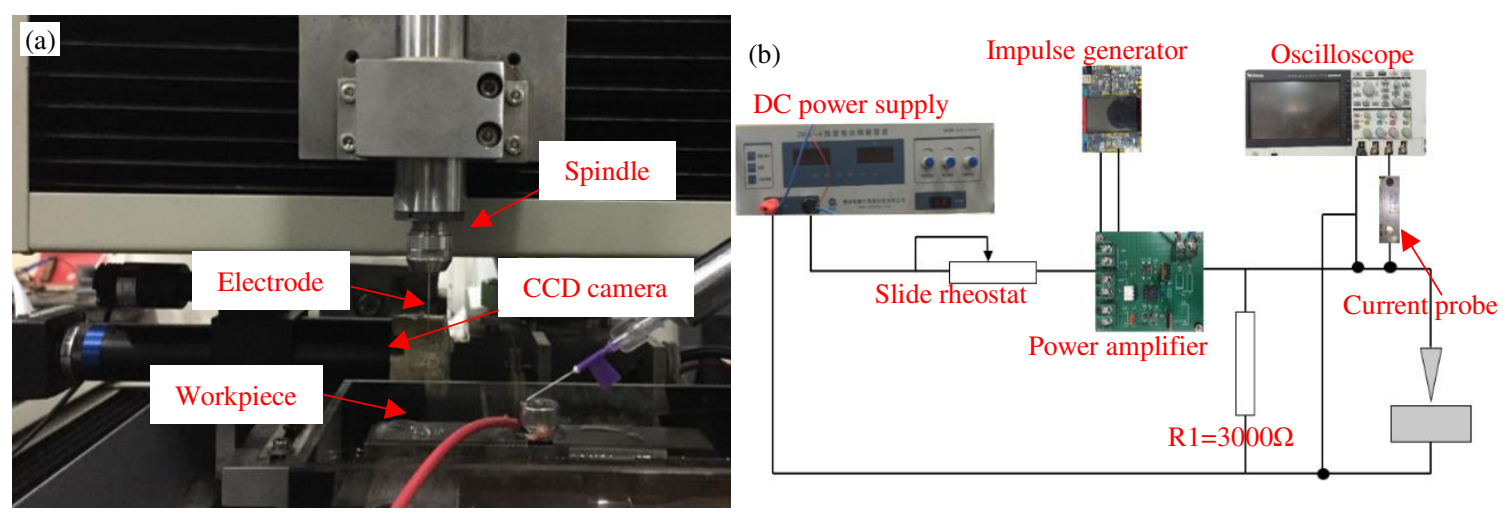

Fig. 1 (a) machine tool and (b) discharge circuit.

\subsection{Electrode and workpiece}

The diameter of the cylindrical tool electrode was $500 \mu \mathrm{m}$ with tungsten as material, and the material for the workpiece was Ti-6Al-4V. The tip of the tool electrode was fine ground by wire electrical discharge grinding (WEDG) method, more details on the fabrication process can be found in our previous research [17]. The surface of the workpiece was ground with 2000 grit emery paper to ensure the original surface roughness had no influence on the experimental 
results.

\subsection{Experimental procedures}

The aim of this section is to investigate the stochastic characteristics of discharge crater size. The three machining parameters, including peak current $\left(i_{p}\right)$, pulse duration $\left(t_{o n}\right)$ and discharge gap width $(d)$ were taken as factors in the design of the experiments. The RSM was used to analyze the influence of the three factors on crater size. The discharge parameters could be adjusted easily, however the determination of the gap width between the electrode and the workpiece is a bit more difficult, hence, a measurement method based on ohmmeter was used. Firstly, the one ends of the tool electrode and workpiece were connected by metal wire, and the other ends were connected by two pins of ohmmeter to measure the resistance $R_{g}$. Then the metal wire was removed and the tool electrode moved down $1 \mu \mathrm{m}$ every step until the resistance value displayed on the ohmmeter was equal to $R_{g}$, and then the gap width was $0 \mu \mathrm{m}$, the tool electrode moved up to a certain distance $d$ afterwards, and the target gap width was obtained. The discharge crater is almost circular or elliptical, hence it is characterized by diameter (for circular crater) and equivalent diameter (the average value of major axis and minor axis for elliptical crater) as shown in Fig. 2.

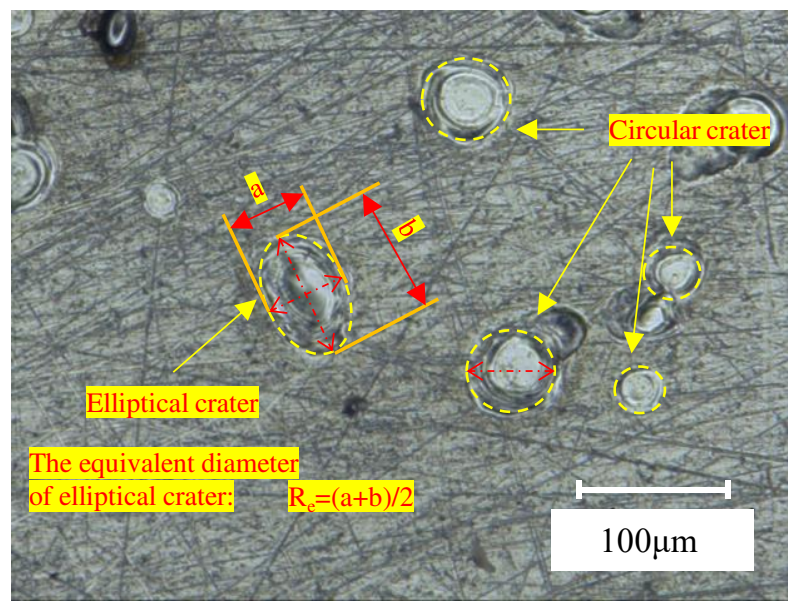

Fig. 2 The two types of discharge crater

Table 1 Experimental conditions

\begin{tabular}{ll}
\hline Items & Descriptions \\
\hline Tool electrode $(-)$ & Tungsten \\
Workpiece $(+)$ & Ti-6Al-4V \\
Open circuit voltage $[\mathrm{V}]$ & 160 \\
Dielectric fluid & EDM oil \\
Electrode diameter $[\mu \mathrm{m}]$ & 500 \\
Pulse duration $[\mu \mathrm{s}]$ & 15 to 75 \\
Discharge current $[\mathrm{A}]$ & 0.5 to 3 \\
Gap width $[\mu \mathrm{m}]$ & 2 to 8 \\
\hline
\end{tabular}

In this study, the finite-successive pulses discharge method was used, and the number of pulses is set to 9.100 discharge craters were obtained under a parameter combination through the repeated experiments, of which the 
equivalent diameters were selected to characterize the size distribution. The experimental parameters are shown in Table 1, and the variable parameters and their levels are listed in Table 2. The experiments were designed based on a central composite design method, and the experimental sequence are shown in Table 3.

Table 2 Variable parameters and their levels

\begin{tabular}{llllll}
\hline Variable parameters & \multicolumn{1}{l}{ levels } & & & & \\
\cline { 2 - 6 } & -1.68179 & -1 & 0 & 1 & 1.68179 \\
\cline { 2 - 6 } $\mathrm{t}_{\mathrm{on}}, \mu \mathrm{s}$ & 15 & 30 & 45 & 60 & 75 \\
$\mathrm{i}_{\mathrm{p}}, \mathrm{A}$ & 0.5 & 1 & 2 & 2.5 & 3 \\
$\mathrm{~d}, \mu \mathrm{m}$ & 2 & 3.5 & 5 & 6.5 & 8 \\
\hline
\end{tabular}

Table 3 Experimental sequence

\begin{tabular}{llll}
\hline No. & $\mathrm{t}_{\mathrm{on}}(\mu \mathrm{s})$ & $\mathrm{i}_{\mathrm{p}}(\mathrm{A})$ & $\mathrm{d}(\mu \mathrm{s})$ \\
\hline $1 \#$ & -1 & 1 & -1 \\
$2 \#$ & 0 & 0 & 0 \\
$3 \#$ & 1 & -1 & 1 \\
$4 \#$ & -1 & 1 & 1 \\
$5 \#$ & 0 & 1.68179 & 0 \\
$6 \#$ & 0 & 0 & 0 \\
$7 \#$ & -1.68179 & 0 & 0 \\
$8 \#$ & 1 & 1 & 1 \\
$9 \#$ & 1 & 1 & -1 \\
$10 \#$ & 1 & -1 & -1 \\
$11 \#$ & 0 & 0 & 0 \\
$12 \#$ & 0 & 0 & -1.68179 \\
$13 \#$ & 1.68179 & 0 & 0 \\
$14 \#$ & 0 & 0 & 0 \\
$15 \#$ & 0 & 0 & 1.68179 \\
$16 \#$ & 0 & -1.68179 & 0 \\
$17 \#$ & 0 & 0 & 0 \\
$18 \#$ & -1 & -1 & 1 \\
$19 \#$ & 0 & 0 & 0 \\
$20 \#$ & -1 & -1 & -1 \\
\hline
\end{tabular}

\subsection{Prediction model of discharge crater size}

The response surface method (RSM) is a statistical test method to optimize stochastic processes. The objective is to find the quantitative law between the test index and each factor and find the best combination at each factor level. When the machining parameters are determined, the size characteristics of discharge craters can be predicted by fitting mathematical models as follows:

$$
Y(o)=a+\sum_{i}^{n=3} b_{i} x_{i}+\sum_{i}^{n=3} c_{i} x_{i}^{2}+\sum_{i<j} d_{i j} x_{i} x_{j}+e
$$


where $Y(o)$ is the target response; $x_{i}$ are coded values of the $i$ th machining process parameters; $a, b_{i}, c_{i}$ and $d_{i j}$ are the regression coefficients; $e$ is the corresponding experimental error of observation; $n$ is the number of variable parameters.

\section{Results and discussions}

\subsection{Characterization of the stochastic characteristic of discharge crater size}

The main types of discharge that produce discharge craters are shown in Fig. 3. Under normal circumstances, the discharge channel takes a short period of time to form after the voltage is applied to the electrode and workpiece, which is called the ignition delay. Fig. 3a shows the normal spark discharge, and differently, the spark discharges shown in Fig. $3 \mathrm{~b}$ last for only a very short time, because the low peak current weakens the maintenance capacity of the discharge channel. In addition, the increase of gap width will also weaken the maintenance capacity of the discharge channel even if the peak current is high as shown in Fig. 3c, the discharge process is terminated before the end of the pulse, and this result is consistent with the study by Li et al. [26]; on the contrary, when the gap width decreases, the electric field strength between the gap increases, and the ignition delay decreases or even disappears, namely arcing as shown in Fig. 3d. It could be obtained from Fig. 3 that the discharge duration varies greatly and it could be a significant reason for the stochastic distribution of the discharge crater size.

Fig. 4a shows the discharge craters, and the diameters of the craters were analyzed with Minitab software. The histogram of diameter size distribution for experiment \#2 is shown in Fig. $4 b$, it can be found that the histogram is inverted bell shaped and roughly symmetrical, hence we intend to use the normal probability density function to fit the distribution of the histogram, and the goodness-of-fit test for the normal distribution was carried out. The probability map of the fit goodness test is shown in Fig. 4c, the median line is the expected percentile of the distribution based on maximum likelihood parameter estimation, and the upper and lower side lines represent the lower and upper limits of the confidence interval for each percentile; the probability map shows that the data points are approximately a straight line and within the confidence interval. The data of group \#2 conform to the normal distribution, because $P$-value satisfies Eq. (2):

$$
P=0.869>\alpha=0.05
$$

where $P$-value (probability value) is the least significant level at which the original hypothesis can be rejected, which is obtained from the sample observations of the test statistic; $\alpha$ is the minimum significance level for rejecting the original hypothesis, and the data can be considered as accord with the normal distribution when $P>\alpha$. Fig. 5 depicts the $P$-values of the goodness-of-fit tests for the 20 sets of experimental data under 20 parameter combinations (as shown in Table 3 ), and except for the 8th group of experimental data, the $P$-values of the other experimental groups are all greater than 0.05 , 
which indicates that the size of the discharge crater obeys normal distribution except for exp. no. \#8. In other words, when the normal probability density function is used to fit the size distribution of the discharge crater, only $5 \%$ of the experimental results have large errors, while the remaining $95 \%$ can be well described using the normal distribution. Therefore, under the condition of accepting a 5\% error, the normal distribution function can be used to characterize the size distribution of discharge craters.
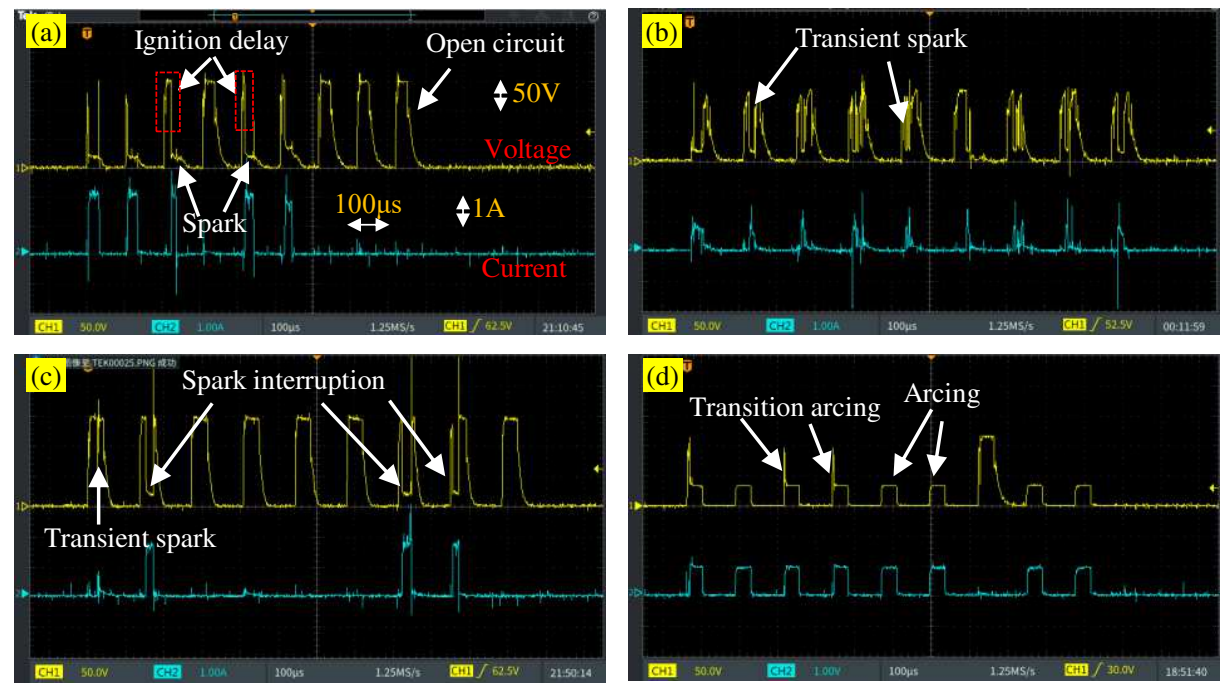

Fig. 3 Discharge waveform obtained under (a) $t_{o n}=45 \mu \mathrm{s}, i_{p}=2 \mathrm{~A}, d=5 \mu \mathrm{m}$, (b) $t_{o n}=45 \mu \mathrm{s}, i_{p}=0.5 \mathrm{~A}, d=5 \mu \mathrm{m}$, (c) $t_{o n}=45 \mu \mathrm{s}, i_{p}=2$ A, $d=8 \mu \mathrm{m}$, and (d) $t_{o n}=30 \mu \mathrm{s}, i_{p}=1 \mathrm{~A}, d=3.5 \mu \mathrm{m}$.

The general equation of normal distribution curve is described as [27]:

$$
f(x)=\frac{1}{\sqrt{2 \pi} \sigma} \exp \left(-\frac{(x-\mu)^{2}}{2 \sigma^{2}}\right)
$$

where $\mu$ is the mean value of the sample; $\sigma$ is the standard deviation of the sample; $x$ is the random variable, i. e. the diameter of discharge crater in the study; $f(x)$ is the probability density of $x$. It can be obtained from Eq. (3) that the shape of normal distribution curve is controlled by $\mu$ and $\sigma$.

(a)

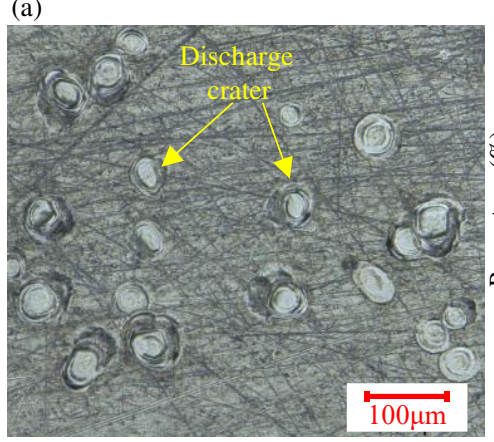

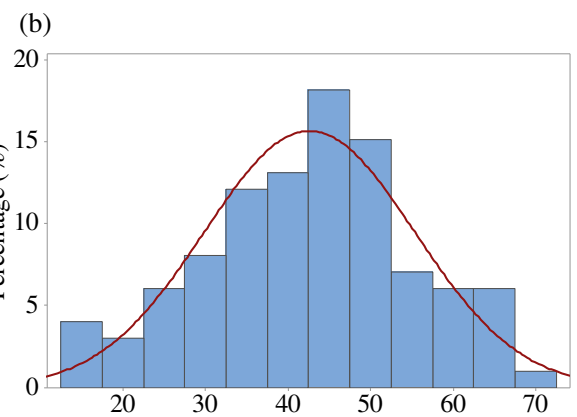

Diameter of discharge craters in Exp.2 $(\mu \mathrm{m})$

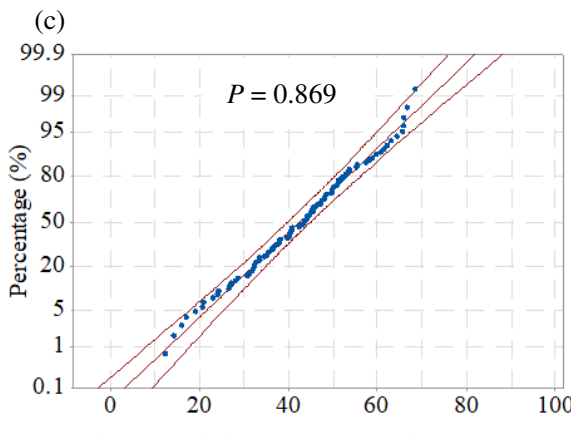

Diameter of discharge craters in Exp.2 $(\mu \mathrm{m})$

Fig. 4 (a) Distribution of discharge craters of exp. 2, (b) histogram of crater diameter distribution and (c) goodness-of-fit test for normal distribution. 


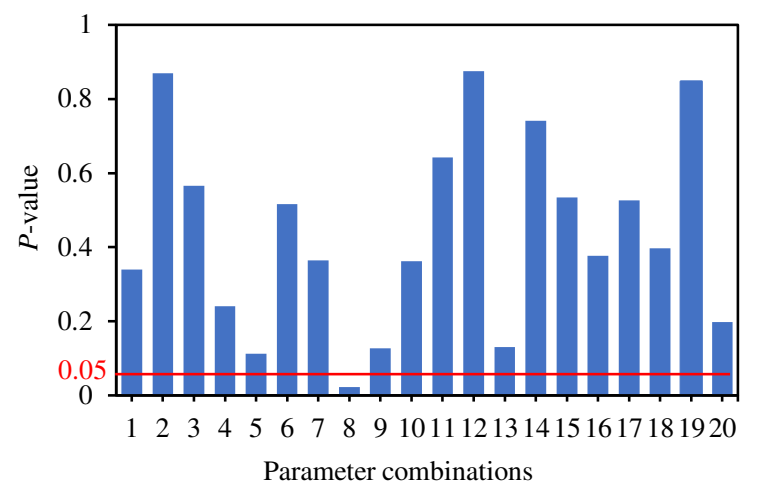

Fig. 5 The $P$-value of goodness-of-fit test for normal distribution of the 20 groups of data.

\subsection{Prediction model for size distribution of discharge crater}

Through the analysis of the previous section, it was found that the stochastic characteristics of discharge crater size could be described by the normal probability density function, hence the mean value and standard deviation of the crater size were used to quantitatively describe the stochastic distribution characteristics of crater size and were taken as the response of RSM. Fig. 6 depicts the output response of the RSM. Across the results, it was obtained that the mean value and standard deviation of crater size are smaller under 3\#, 10\#, 16\#, 18\# and 20\# parameter combinations, in which the current levels are low or gap width levels are high, hence discharge current and gap width have a significant influence on the crater size, and the influence of pulse width on crater size is relatively small.
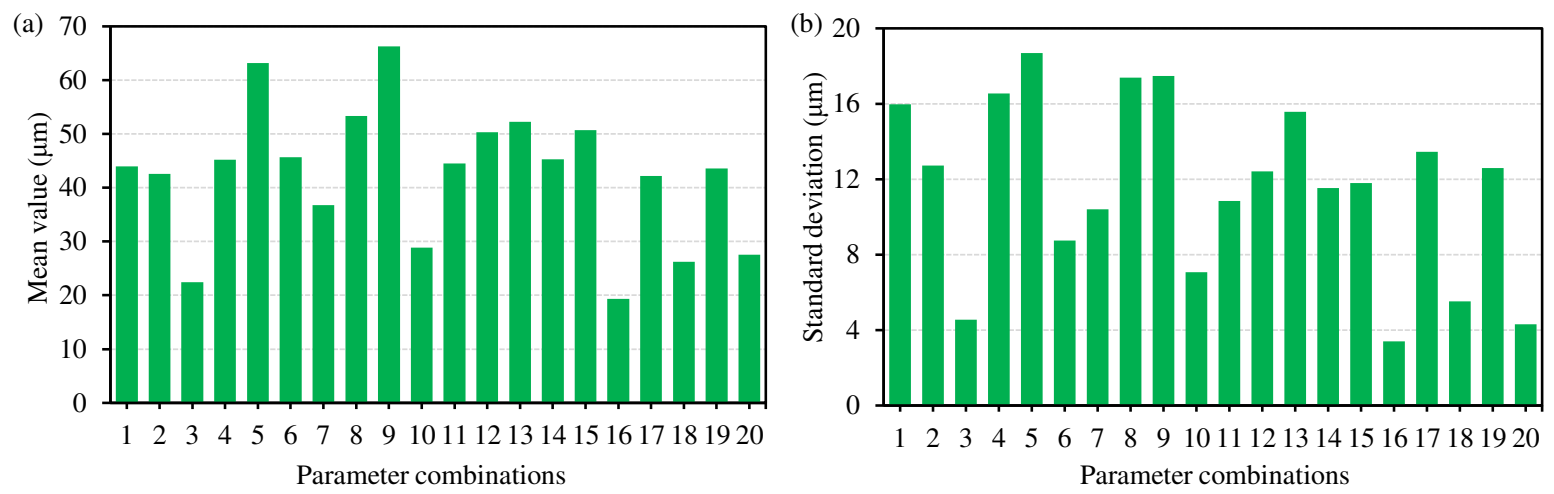

Fig. 6 Mean value and standard deviation of crater size under different parameter combinations.

A second order polynomial is used to clarify the relationship between machining parameters and the size distribution of discharge craters, as shown in Eqs. (4), (5).

$$
\begin{aligned}
Y_{1}=44.17+ & 3.95 x_{1}+12.99 x_{2}-1.38 x_{3}-1.13 x_{1}^{2}-2.28 x_{2}^{2}+0.98 x_{3}^{2}+4.13 x_{1} x_{2}-2.42 x_{1} x_{3}-0.49 x_{2} x_{3} \\
Y_{2}= & 11.687+0.939 x_{1}+5.246 x_{2}-0.137 x_{3}-0.268 x_{1}^{2}-0.421 x_{2}^{2}+0.042 x_{3}^{2}+0.070 x_{1} x_{2} \\
& -0.548 x_{1} x_{3}-0.220 x_{2} x_{3}
\end{aligned}
$$

Where $Y_{1}$ is the mean value; $Y_{2}$ is standard deviation; $x_{1}, x_{2}$ and $x_{3}$ are pulse width, peak current and discharge gap width respectively. 
In order to verify the validity of the prediction model given in Eqs. (4) and (5), the test experiment was conducted as shown in Table 4. The results of the test experiment and the prediction are shown in Fig. 7, where MV1 and MV2 are the mean values of crater diameter obtained from the prediction model and the test experiment respectively; SD1 and SD2 are the standard deviation of crater diameter obtained from the prediction model and the test experiment respectively. The maximum errors for the predictive values of mean value and standard deviation are $5.69 \%$ and $6.71 \%$ respectively, and the average errors for the two are $4.29 \%$ and $5.49 \%$ respectively. Thus, the prediction errors are acceptable, indicating that the prediction model is effective.

Table 4 Test experiment

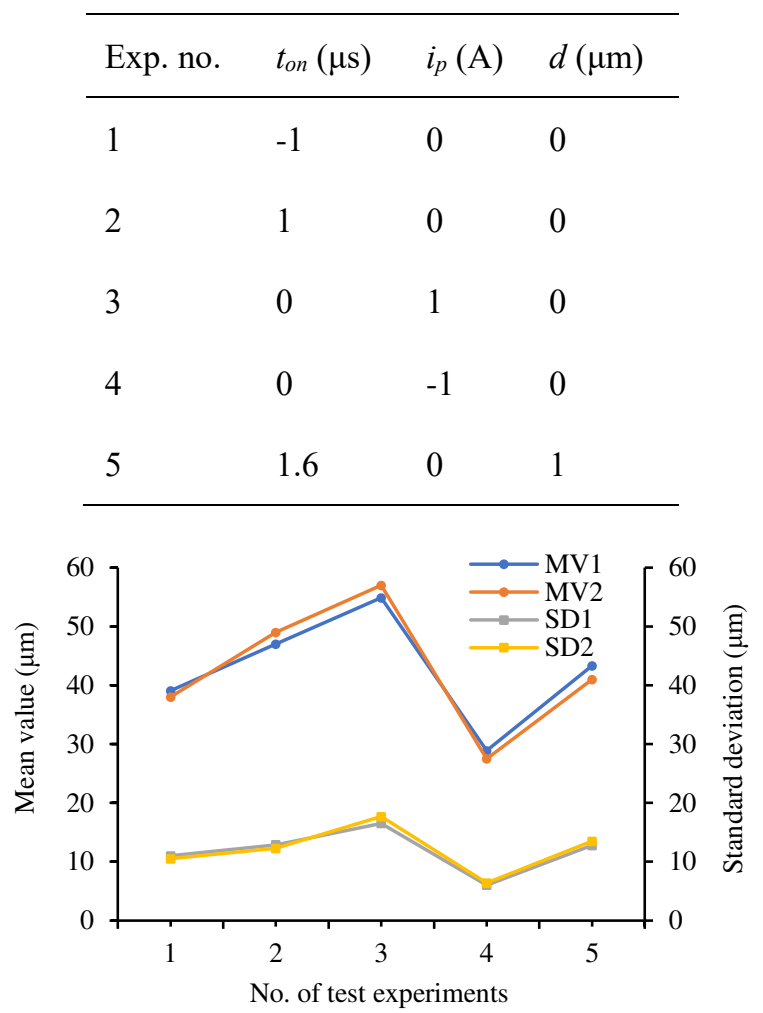

Fig. 7 Experimental and predictive results for crater size

\subsection{Effects of machining parameters on the discharge crater size distribution}

The crater size is related to the discharge energy distributed to the surface of the workpiece, which can be calculated as [11]:

$$
E_{\mathrm{w}}=\eta E=\eta u i t
$$

where $E_{W}$ is the discharge energy distributed to the surface of the workpiece, $\eta$ is the energy distribution coefficient, $E$ is the discharge energy, and $u, i$ and $t$ are average discharge voltage, average discharge current and discharge duration respectively. Generally, $u$ is regarded as a constant, and $i$ increases with the increase of peak current during discharge process. The larger the $E_{W}$ is, the larger the crater size is. Therefore, the discharge energy can be described by the crater 
size. The effects of pulse width, peak current and discharge gap width on the response, i. e. mean value and standard deviation of discharge crater diameter, have been investigated with contour map obtained by RSM as follows:

(1) Effects of parameters on the mean value of discharge crater diameter

Fig. 8 shows the interaction of pulse width and peak current on the mean value. It is obtained that the mean value increases with the increase of pulse width and peak current, and that the sensitivity of mean value to peak current increases under the high level of pulse width, that is because the discharge energy in a single pulse increases with the increase of peak current, which results in an increase of the discharge crater size. However, the pulse width has little effect on the average size of the discharge crater at low peak current level, this is due to the maintenance ability of the discharge channel decreases when the peak current is low, and the discharge channel is easily extinguished while the pulse is still on, as shown in Fig. 3b. Under this circumstance, the discharge duration $t$ and discharge energy will not increase with the increase of pulse width. Under the low level of pulse width, the mean value increases slightly with the increase of peak current, and the sensitivity of mean value to peak current is very small, that is because the small pulse width limits the increase of discharge energy $E$ in a single pulse.

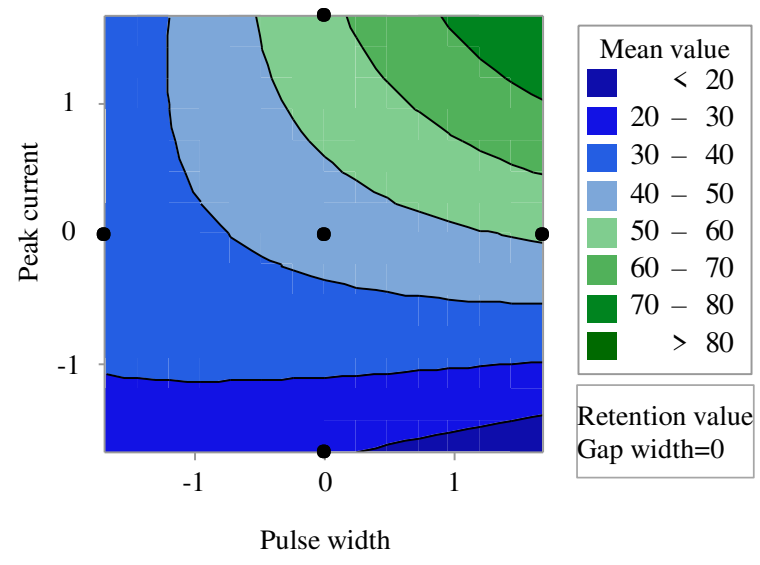

Fig. 8 Contour plot for mean value vs $i_{p}$ and $d$

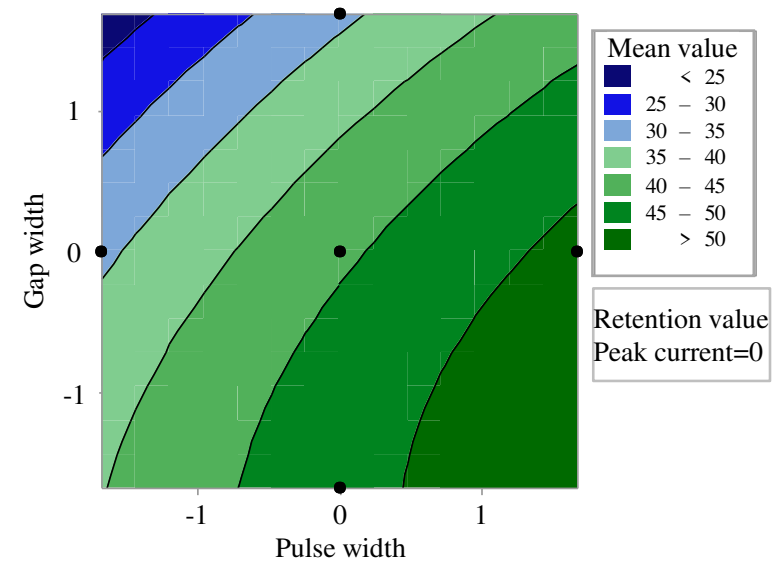

Fig. 9 Contour plot for mean value vs $d$ and $t$

Fig. 9 shows the effect of the interaction of pulse width and gap width on the mean value. It has been observed that the mean value is minimum with the combination of high level gap width and low level pulse width, and the mean value is maximum with the combination of low level gap width and high level pulse width. The influence of gap width on mean value can be considered from two aspects: firstly, with the increase of the gap width, the energy distribution coefficient $\eta$ decreases [28], and the discharge channel expands and the heat flux decreases [29], which will cause the decrease of $E_{W}$; secondly, the increase of the gap width will lead to the instability of the discharge channel and the weakening of the discharge maintenance ability; according to the comparison between Fig. 3a and Fig. 3b, increasing the gap width will make the discharge channel easier to extinguish within the pulse duration when other conditions remain unchanged.

(2) Effects of parameters on the standard deviation of discharge crater diameter 
Standard deviation is a measure of the dispersion for a group of data. A large standard deviation represents a large difference between most discharge crater sizes and their mean value, and a smaller standard deviation indicates that these sizes are closer to the mean value. Fig. 10 shows the influences of pulse width and peak current on standard deviation; the sensitivity of standard deviation to pulse width is low, but it is high to peak current. The standard deviation increases with the increase of peak current, that is because the discharge power increases with the increase of peak current, and the discharge duration $t$ is random even if the processing parameters are the same; therefore, when $i$ increases, a small change of $t$ will lead to a significant change of $E$ as shown in Eq. (6), which leads to the increase of the size difference of discharge craters. On the other hand, according to Fig. 3, the relationship between discharge duration $t$ and pulse width $t_{\text {on }}$ can be obtained as follows:

$$
0 \leq t \leq \text { ton }
$$

Where $t=0$ means that it is an open circuit pulse, $t=t_{\text {on }}$ means that there is no ignition delay and the discharge channel does not distinguish during the pulse duration. When $\mathrm{t}_{\mathrm{on}}$ increases, the value range of $\mathrm{t}$ also increases, which leads to the further increase of the dispersion of discharge energy $E$. Therefore, the maximum standard deviation appears in the combination of high-level current and high-level pulse width.

It can be seen from Fig. 11 that the influence of gap width on standard deviation increases with the increase of pulse width, this is due to the discharge duration $t$ tends to be more consistent under the low-level pulse width and gap width, and with the increase of gap width, the maintenance ability of the discharge channel decreases and it is more likely to be extinguished, which will increase the dispersion of the crater size. At the circumstance of high-level pulse width, the influence of gap width on standard deviation increases, which is also related to the maintenance ability of the discharge channel. The maintenance ability of the discharge channel decreases with the increase of discharge gap width, which leads to a greater dispersion of discharge duration $t$ and the discharge energy $E$ in a single pulse.

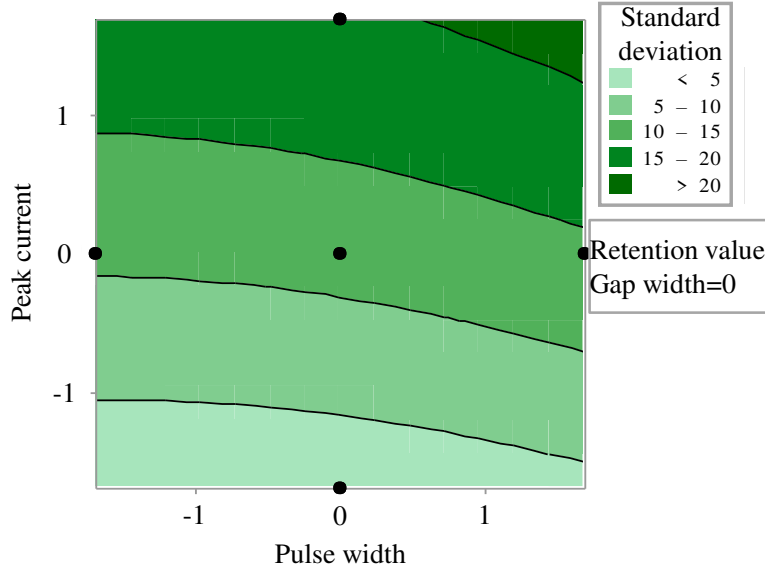

Fig. 10 Contour plot for standard deviation vs $i_{p}$ and $t_{p}$

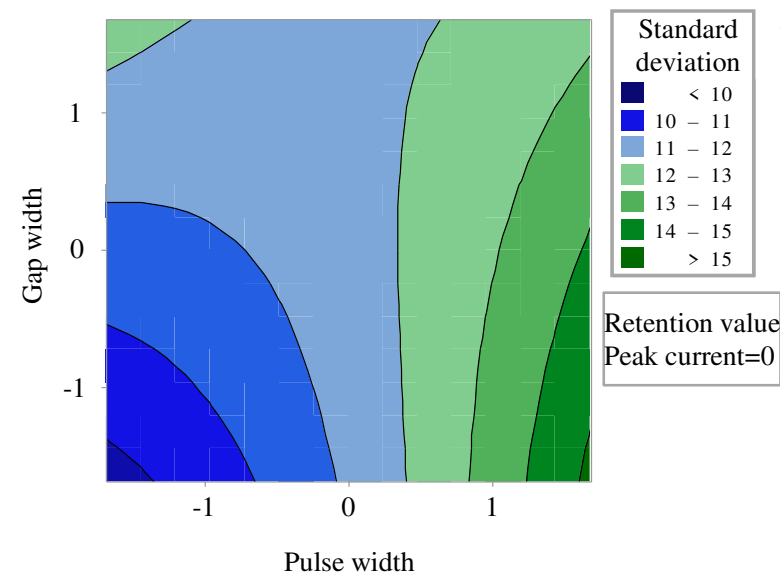

Fig. 11 Contour plot for standard deviation vs $d$ and $t_{p}$ 


\section{Simulation model for micro-EDM}

EDM is an important method for manufacturing superhydrophobic surfaces, surface enhanced Raman scattering and other functional surfaces. Surface morphology plays an important role in these functions. In order to accurately manufacture these functional surfaces, it is necessary to predict the surface morphology, hence a simulation model of the EDM process was developed. In this model, the meshing method is similar to the models of Zhang's [30] and Jeong's [31]: The electrode and workpiece are divided into squares of the same size as shown in Fig. 12.

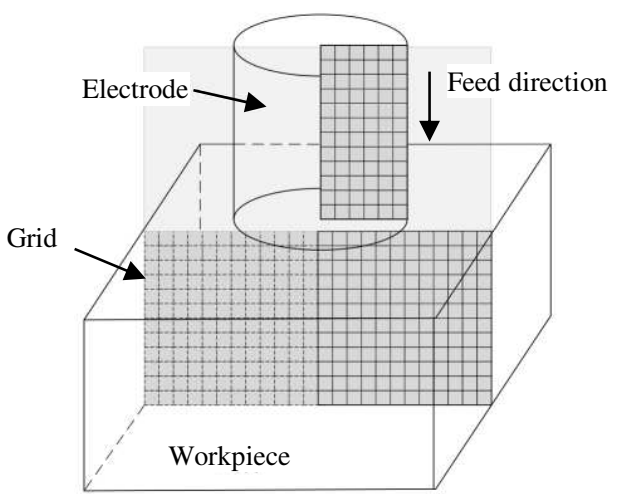

Fig. 12 The 2D simulation model of EDM milling process

The model proposed in this study is based on the following assumptions:

(1) There is only one discharge occurs in every time step, the time step is described as Eq. (8)

$$
\Delta t=1 / f
$$

Where $\Delta t$ is the time step, $f$ is the pulse frequency.

(2) Only spark discharge is considered, and the spark discharge occurs in the shortest gap width between the two points of the electrode and the workpiece.

(3) The stochastic distribution of the crater size is considered, and size distribution characteristics are obtained according to the experiment.

(4) The diameter of the plasma channel is not considered, and the discharge energy is delivered point to point.

(5) The shape of the discharge crater is cone-shaped, and the discharge debris is ignored.

The model includes horizontal feed and vertical feed, and the flow chart of the simulation process is shown in Fig. 13. 


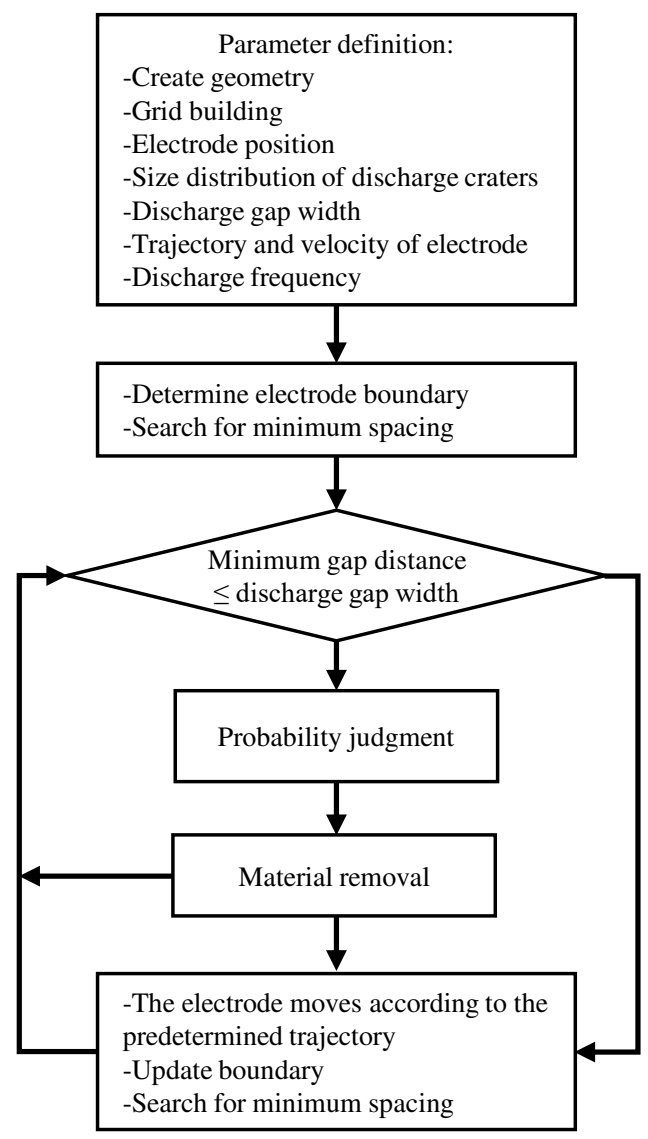

Fig. 13 Flow chart of the simulation model

The electrode and workpiece are represented by black square pixels, the gap is represented by white pixels, and each pixel has three indexes, i.e. abscissa, ordinate and gray values. The positions of the electrode and workpiece are determined by the abscissa and ordinate, and the contour of the electrode and workpiece is judged by the gray value. The workpiece and the electrode are in the same set of absolute coordinates. In Zhang's model, a fixed size of the discharge crater is used to simulate the EDM process so as to simplify the calculation. This method can obtain certain details such as material removal rate, electrode wear rate and electrode shape, but it is difficult to simulate the surface morphology of EDM because in actual machining, the size of the discharge craters varies greatly even if using the same parameter combination, as mentioned above. Therefore, assumption (3) can in theory make the model closer to the actual EDM surface morphology in theory.

\subsection{Searching for discharge position}

In order to simplify the calculation, the lower boundary of the electrode and the upper boundary of the workpiece are determined first, and the point pair from the two boundaries separately with the minimum distance is searched then. When there are many point pairs that meet the requirements, one of which is randomly selected as the discharge position. The determination method of discharge position is calculated according to Eq. (9) [30]:

$$
d^{2}=\left(x_{i, e}-x_{j, w}\right)^{2}+\left(y_{i, e}-y_{j, w}\right)^{2} \leq D^{2}
$$


where $d$ is the distance between the two points of the electrode and workpiece, $\quad\left(x_{i, e}, y_{i, e}\right)$ is the coordinates of any point on the electrode contour, $\left(x_{j, w}, y_{j, w}\right)$ is the coordinates of any point on the workpiece contour, and $D$ is the set discharge gap width.

\subsection{Material removal}

The actual crater (shown in Fig. 14a) is shaped like a spherical cap as shown in Fig. 14b, and it can be simplified to a geometric model as shown in Fig. 14c, where $R$ is the diameter of the bulge, $h$ is the depth between the top and bottom of the crater, and $\bar{R}$ and $\bar{h}$ are the average values of $R$ and $h$ respectively. Through calculation, it can be obtained that $\bar{R} / \bar{h}$ is 10 approximately.
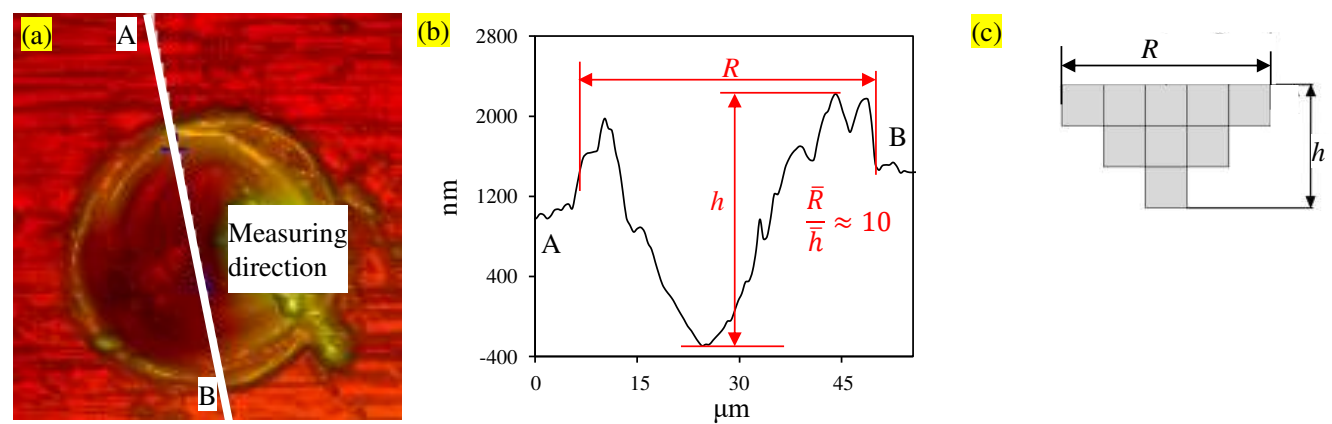

Fig. 14 The morphology of discharge crater: (a) AFM photo, (b) cross section and (c) Equivalent geometric model of crater.

Due to the discharge crater on the electrode being not easy to measure, the discharge crater on the electrode can be obtained according to the Eq. (10) [30]:

$$
r=r^{\prime} \theta^{1 / 3}
$$

where $r$ and $r^{\prime}$ are the crater diameters on electrode and workpiece separately; $\theta$ is the electrode wear rate, and it is obtained by experiment. In this model, the size of the crater diameter is normally distributed, and the normal probability density function is divided into 11 rectangles according to the Riemann integral in order to generate the discharge crater with normal distribution probability. The ordinate of each rectangle represents the probability of its occurrence, and the abscissa represents the crater diameter under this probability. The flow chart for judging discharge crater size is shown in Fig. 15 . 


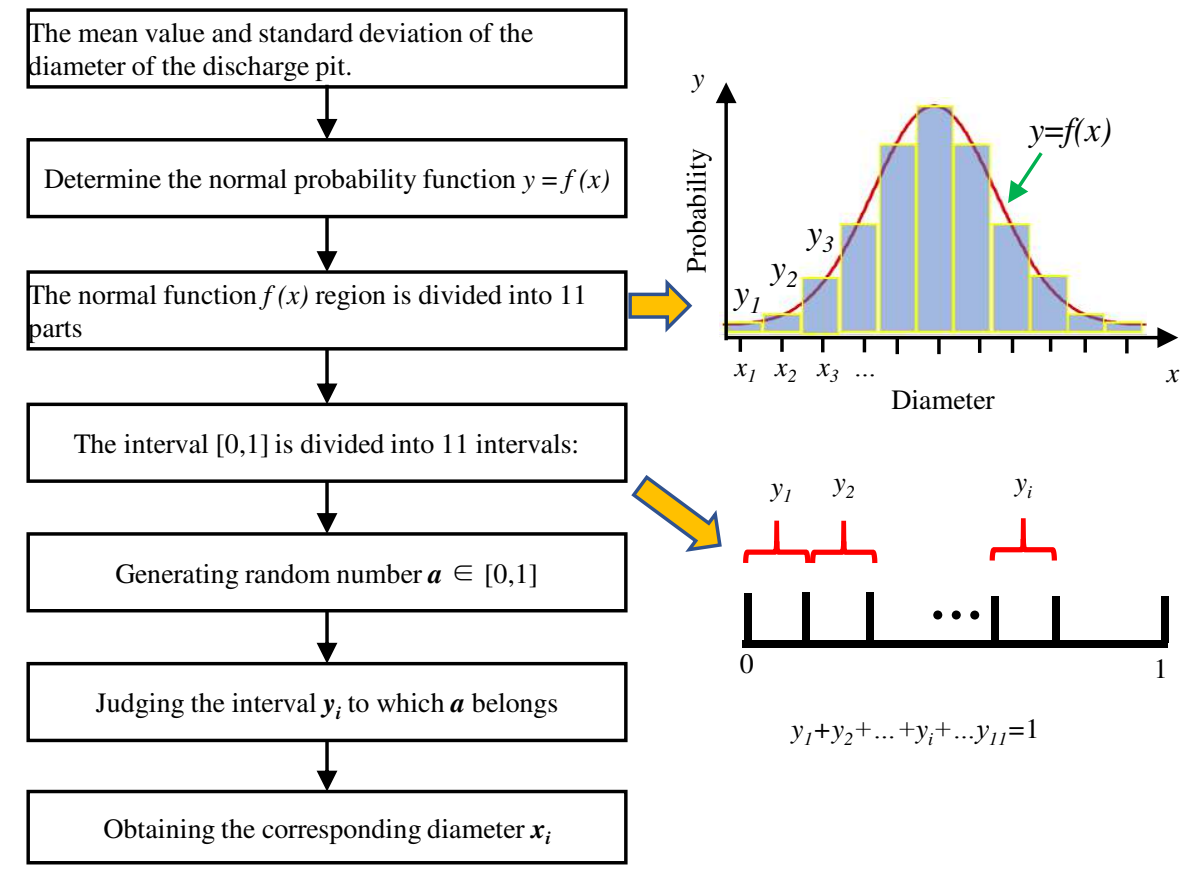

Fig. 15 Flow chart of judging discharge crater size

\subsection{The forming of EDM surface morphology using simulation}

In order to demonstrate the formation of EDM surface, the simulation research is carried out, and the simulation parameters are shown in Table 5.

Table 5 Simulation parameters

\begin{tabular}{llll}
\hline Item & Unit & Value & Value \\
\cline { 2 - 4 } & & MSDS & MFS \\
\hline Grid size & $\mu \mathrm{m}$ & 0.2 & 0.2 \\
Electrode diameter & $\mu \mathrm{m}$ & 500 & 500 \\
Standard deviation of crater & $\mu \mathrm{m}$ & 3.4 & $\backslash$ \\
Mean value of crater on workpiece & $\mu \mathrm{m}$ & 19.4 & 19.4 \\
Pulse frequency & $\mathrm{Hz}$ & 12500 & 12500 \\
Tool wear ratio & $\backslash$ & $16.3 \%$ & $16.3 \%$ \\
Discharge gap width & $\mu \mathrm{m}$ & 12 & 12 \\
Electrode feed rate & $\mu \mathrm{m} / \mathrm{s}$ & 1 & 1 \\
Depth/diameter ratio of crater & $\backslash$ & 0.1 & 0.1 \\
\hline
\end{tabular}

In the existing geometry simulation, the discharge crater is set to a fixed size, in order to investigate the influence of normal distributed crater size on the simulation results, two models, the model based on stochastic distribution of crater size (MSDS) and the model based on fixed crater size (MFS), were developed. The simulation processes of the two models are shown in Fig. 16 and Fig. 17 separately. In the simulation, the first discharge location is stochastic, and the first overlap occurs at the $9^{\text {th }}$ spark in MSDS, and the first overlap occurs at the $6^{\text {th }}$ spark in MFS; with the increase of the number of sparks, the number of overlapping craters gradually increases until the surface of the workpiece is completely 
covered by craters as shown in Fig. 16c and Fig. 17c. Because the size of the electrode and workpiece is much larger than the crater size, some details cannot be shown in Fig. 16 and Fig. 17, hence the EDM surface morphology data for Fig. 16c and Fig. 17c, were extracted and the broken line diagram was drawn in Fig. 16d and Fig. 17d separately. It is found that the simulated surface of the two models is significantly different by comparing Fig. 16d and Fig. 17d, the degree of the surface undulation of MSDS is larger compared with that of MFS, and the simulated surface of MFS is regularly wavy; this is because the crater size in MSDS shows considerable differences, and the difference in depth of crater causes the fluctuation of surface height.
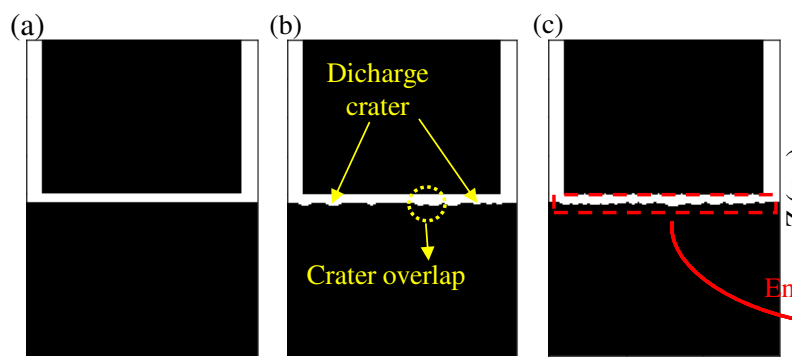

(d)

Fig. 16 Evolution of surface morphology of MSDS
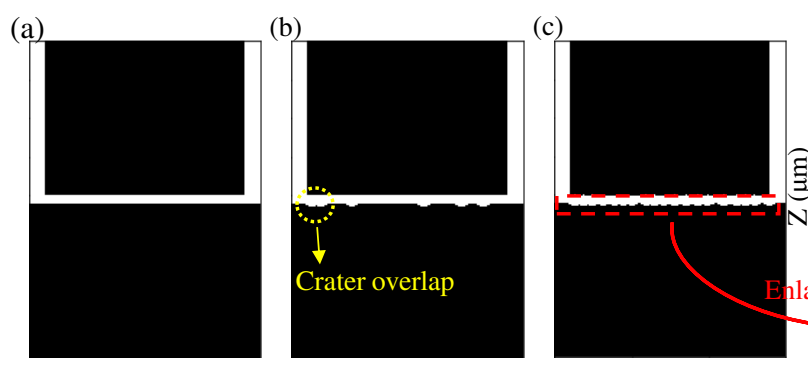

(d)

Fig. 17 Evolution of surface morphology of MFS

Mohammad et al. [32] thought the traditional roughness $(\mathrm{Ra})$ could not accurately describe the surface morphology characteristics of EDM. They machined two groups of surfaces with the same $R a$, but with different characteristics of discharge craters. This is because $R a$ primarily focuses on the characteristics of the morphology in the direction perpendicular to the surface, but does not pay attention to the morphological characteristics parallel to the surface. Currently, most of the existing EDM surface morphology models only focus on the morphology features perpendicular to the surface. Therefore, in order to describe the characteristics of EDM surface morphology more accurately and compare the applicability of the two surface morphology prediction models, the surface height and adjacent peaks interval distribution are used to describe the surface morphology features of the two surface morphology prediction models. As shown in Fig. 18, in the schematic diagram of cross section morphology, the height of the lowest point A is defined as 0 , $L_{i}$ and $H_{i}$ are the interval between adjacent peaks and the height of the data point respectively. It should be noted that using this calculation method, the mean value of surface height is equal to the roughness $R a$. 


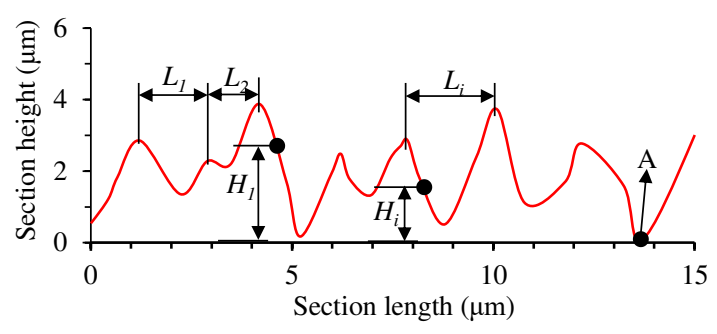

Fig. 18 The schematic diagram of cross section morphology

Fig. 19 shows the surface height and peak interval of the simulated surfaces. As shown in Fig. 19a and b, it can be seen that the surface height and the distance of adjacent peaks are normally distributed on the simulated surface of MSDS. As for MFS, the surface height and the distance of adjacent peaks are irregularly distributed as shown in Fig. 19c and d. From Fig. 19c, it can be observed that the surface height of MFS is relatively concentrated, which indicates that the surface is relatively flat. Fig. 19d shows that the surface peak interval is about $25 \mu \mathrm{m}$, compared with Fig. 19b, it can be seen that, the simulated surface of MFS is more regular that of MSDS, this feature can also be obtained by comparing Fig. 16d and Fig. 17d.
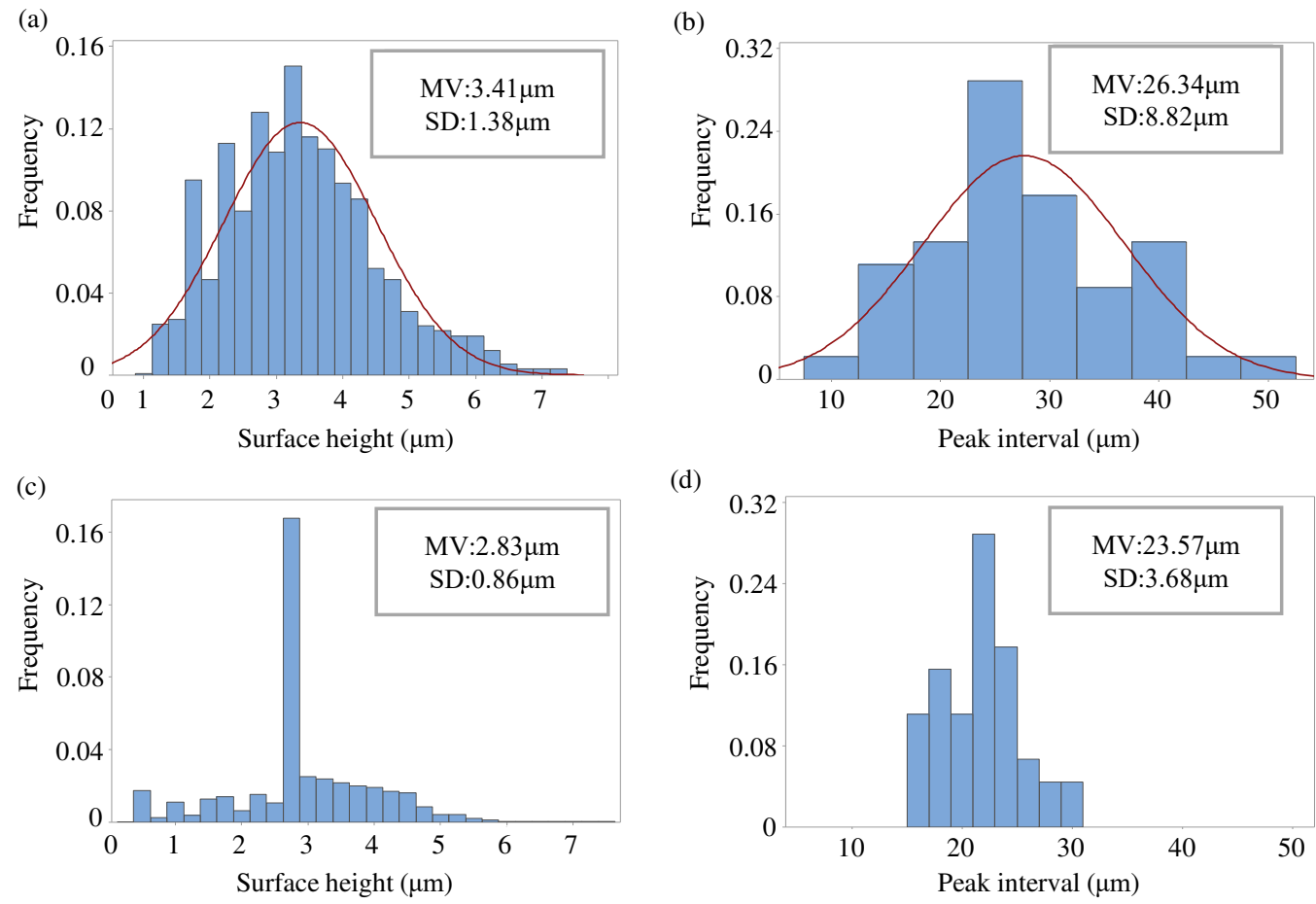

Fig. 19 The distribution characteristics of (a) (c) the surface height and (b) (d) interval of adjacent peaks for MSDS and MFS separately.

\section{Validation experiment for the model}

In order to verify the effectiveness of the surface morphology prediction model, a verification experiment was carried out, and the machining parameter combination adopted are \#2. The experimental result is shown in Fig. 20a, and the data of surface morphology were obtained by laser confocal microscope. Because the EDM surface is isotropic, the surface 
profile data of $\mathrm{y}=300 \mu \mathrm{m}$ section were selected for analysis as shown in Fig. 20b. The surface height and adjacent peaks' distance characteristics of the machined surface are shown in Fig. 21, the Surface height and peak interval are normal distributions. By comparing Fig. 19 and Fig. 21, it can be seen that the simulated surface of MSDS has some characteristics similar to that of the machined surface, while the simulated surface of MFS does not have these characteristics. It is observed from Fig. 20b that the fluctuation of the machined surface is obvious, and compared with Fig. 16d and Fig. 17d, we can conclude that the simulated result of MSDS is closer to the experimental result than that of MFS.

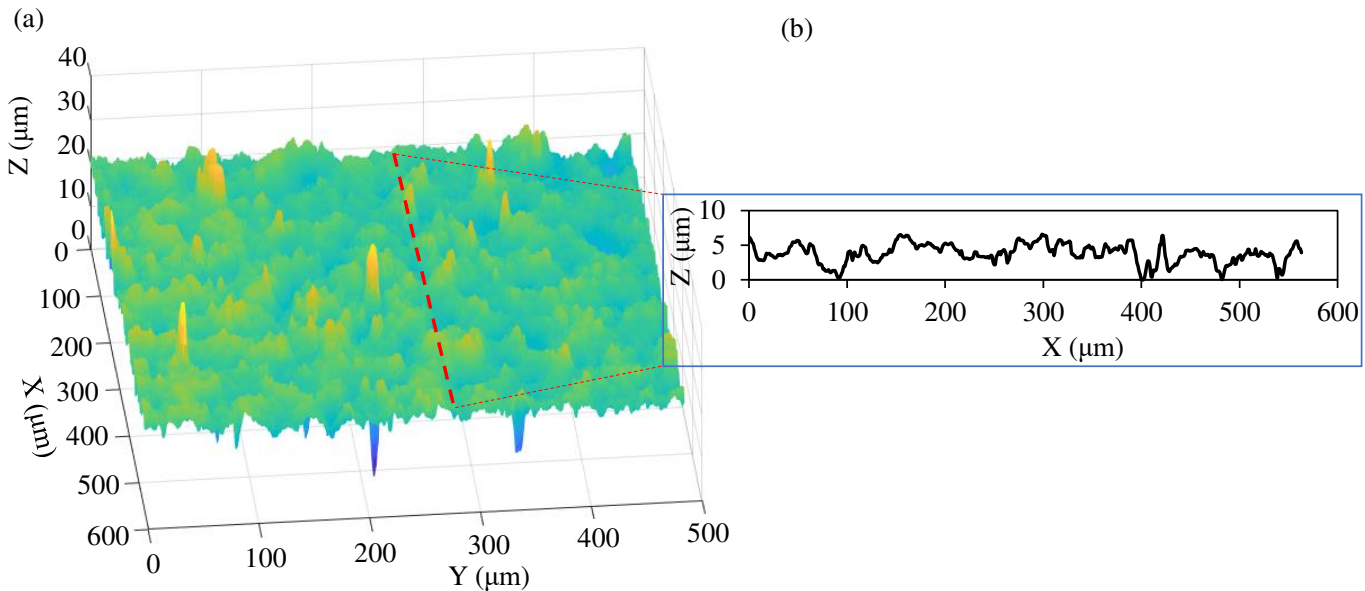

Fig. 20 The (a) 3D Morphology and (b) 2D Morphology of the machined surface.
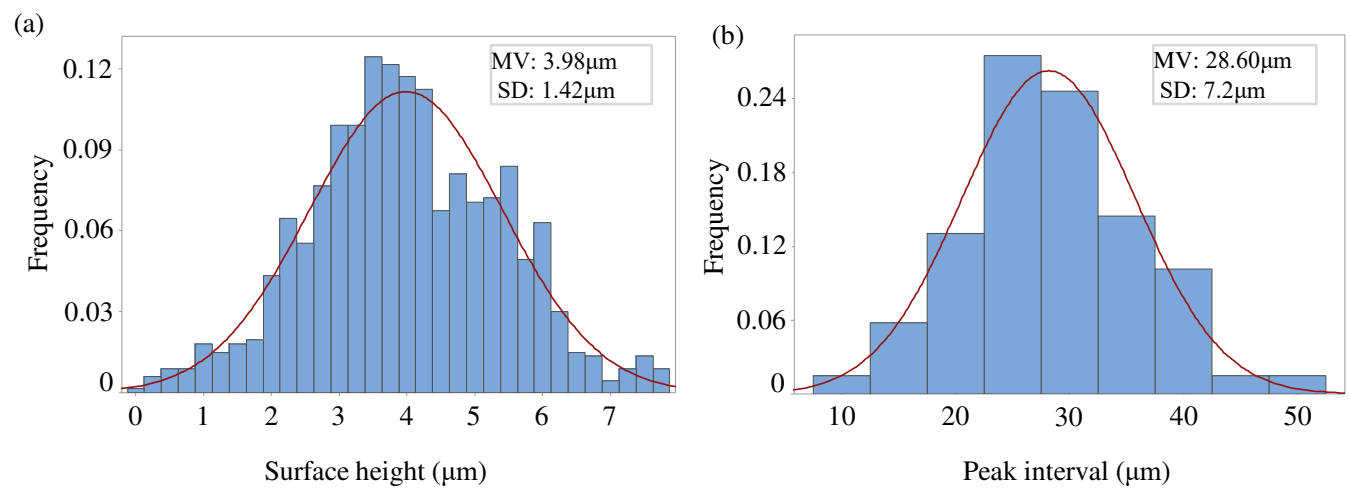

Fig. 21 The distribution characteristics of the surface height and interval of adjacent peaks for machined surface As shown in Fig. 22a, MV'1, MV'2 and MV'3 are the mean values of surface height for the results of MSDS, MFS and experiment separately; SD'1, SD'2 and SD'3 are the standard deviation of surface height for the results of MSDS, MFS and experiment separately. It could be obtained that the simulated surface of MSDS is closer to the machined surface, SD'2 is less than SD'1 and SD'3, which indicates that the surface height of MFS is more consistent, this can also be seen in Fig. 19c. Fig. 22b shows the characteristic of adjacent peak intervals for simulated surfaces and machined surface, where MV"1, MV"2 and MV"3 are the mean values of peak interval for the results of MSDS, MFS and experiment separately, SD"1, SD"2 and SD"3 are the standard deviation of peak interval for the results of MSDS, MFS and 
experiment separately. It can be seen that the disparity between MV"1, MV"2 and MV"3 is less distinctive, while SD"2 is lower than SD"1 and SD"3, which indicates that the peak interval for the simulated surface of MFS is more consistent. The peak interval of the machined surface depends on the size of the discharge crater. Therefore, we compared the average peak interval with the average diameter of the craters, and obtained that the average diameter of the discharge crater is about 1.6 times the average peak interval in the calculation.

The average prediction errors of MSDS and MFS are listed in Table 6, it can be seen that the prediction errors of MV' $(R a)$ of the two models are almost the same while the other prediction errors are quite different, this is because the average surface height of the two models is almost the same, although the surface morphology characteristics are different. As shown in Table 6, the overall average prediction error is defined as:

$$
e_{o}=\left(e_{M V^{\prime}}+e_{s d^{\prime}}+e_{M V^{\prime \prime}}+e_{S D^{\prime \prime}}\right) / 4
$$

where $e_{M V^{\prime}}$ and $e_{S D^{\prime}}$, are average prediction error for mean value $\left(\mathrm{MV}^{\prime}\right)$ and standard deviation (SD') of surface height respectively, $e_{M V^{\prime \prime}}$ and $e_{S D^{\prime \prime}}$ are average prediction error for mean value and standard deviation of peak intervals respectively, $e_{o}$ is the average of overall prediction error. As shown in Table 6, it can be seen that MSDS has higher prediction accuracy.
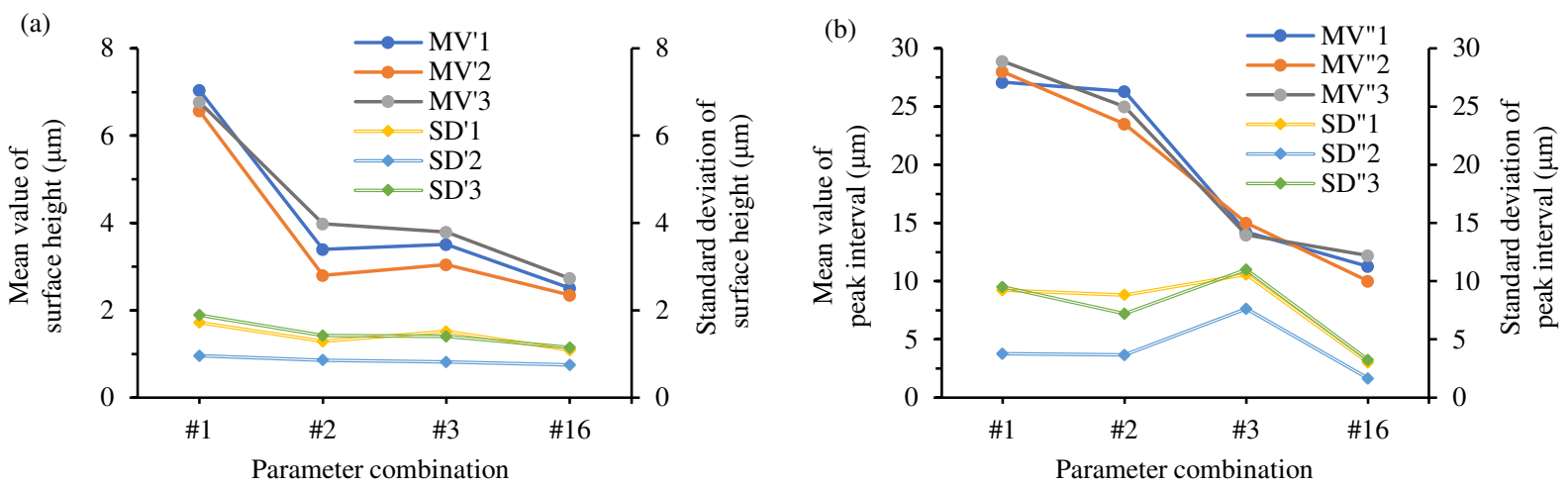

Fig. 22 Comparison for (a) surface height and (b) peak interval of the simulated surface and the machined surface.

Table 6 Comparison of the average prediction error of the two simulation models.

\begin{tabular}{llllll}
\hline Model type & \multicolumn{2}{l}{$\%$ of the average prediction error } & $\begin{array}{l}\text { \% of overall } \\
\text { average error }\end{array}$ \\
\cline { 2 - 6 } & $\mathrm{MV}^{\prime}(R a)$ & $\mathrm{SD}^{\prime}$ & $\mathrm{MV}^{\prime \prime}$ & $\mathrm{SD}^{\prime \prime}$ \\
\hline MSDS & 7.50 & 7.98 & 5.21 & 8.89 & 7.39 \\
\hline MFS & 8.51 & 41.21 & 8.61 & 47.14 & 26.37 \\
\hline
\end{tabular}

\section{Conclusions}

In this study, the stochastic characteristics of the discharge crater size were characterized by mathematical probability models, the causes and the influencing factors of the stochastic characteristic were analyzed. A surface morphology 
prediction model based on the stochastic characteristics of crater size was developed. By comparing with the existing simulation methods, the relationship between the randomness of discharge pit size and surface morphology was explored. The main conclusions are as follows:

1. The normal distribution probability density function is used to fit the crater size under 20 different parameter combinations, and 19 groups of results have passed the goodness-of-fit test, it is considered that the crater size conforms to the normal distribution under the condition of accepting $5 \%$ error rate. The main reason for the different size of the discharge crater is that the discharge durations are different in discharge pulses, which will cause the different discharge energy.

2. The average crater size increases with the increase of pulse width when the peak current is large, while the influence of pulse width on the average crater size decreases when the peak current is small. This is because the maintenance capability of the discharge channel decreases with the decrease of the peak current, and discharge channel is easily interrupted, resulting in the discharge energy not increasing with the increase of pulse width. The increase of gap width will also lead to the weakening of the maintenance ability of discharge channel with other parameters unchanged. The standard deviation of the crater size increases with the increase of the pulse width and decrease of the maintenance capability of the discharge channel.

3. The surface height and adjacent peak interval of the machined surface are normally distributed, and these characteristics could be simulated by MSDS (the model based on stochastic distribution of crater size), while MFS (the model based on fixed crater size) could not simulate the distribution characteristics of surface height and peak interval. The peak interval is influenced by crater size, and the average diameter of crater is about 1.6 times the average peak interval under the experimental conditions of this study.

4. The prediction errors for the surface roughness of MSDS and MFS are $7.50 \%$ and $8.51 \%$ respectively while the overall average errors for the two models are $7.65 \%$ and $26.62 \%$ respectively, which indicates that MSDS has higher simulation accuracy.

Autuor contribution Shaojie Hou: conceptualization, methodology, writing - original draft preparation, investigation, data curation, visualization. Jicheng Bai: writing - review, editing, supervision, project administration, funding acquisition.

Funding This work is supported by the National Natural Science Foundation of China (No. 51575137), the Key Project of Natural Science Foundation of Heilongjiang Province of China (No. ZD2019E005), and the National Natural Science 
Foundation of China (No. 51975156).

Availability of data and material Not applicable.

Code availability Not applicable.

\section{Declarations}

Ethical approval Not applicable.

Consent to participate Not applicable.

Consent for publication All authors have read and agreed to the published version of the manuscript.

Conflict of interest The authors declare no competing interests.

\section{References:}

1. Ho KH, Newman ST (2003) State of the art electrical discharge machining (EDM). Int J Mach Tools Manuf 43 (13):1287-1300

2. Ezugwu EO, Wang ZM (1997) Titanium alloys and their machinability—a review. J Mater Process Technol 68 (3):262-274

3. Klocke F, Schwade M, Klink A, Kopp A (2011) EDM Machining Capabilities of Magnesium (Mg) Alloy WE43 for Medical Applications. Procedia Eng 19:190-195

4. Li CJ, Li Y, Tong H, Zhao L, Kong QC, Wang ZQ (2016) An EDM pulse power generator and its feasible experiments for drilling film cooling holes. Int J Adv Manuf Technol 87 (5-8):1813-1821

5. Azad MS, Puri AB (2012) Simultaneous optimisation of multiple performance characteristics in micro-EDM drilling of titanium alloy. Int J Adv Manuf Technol 61 (9-12):1231-1239

6. Krishna Kiran MPS, Joshi SS (2007) Modeling of Surface Roughness and the Role of Debris in Micro-EDM. J Manuf Sci Eng 129 (2):265-273

7. Salonitis K, Stournaras A, Stavropoulos P, Chryssolouris G (2009) Thermal modeling of the material removal rate and surface roughness for die-sinking EDM. Int J Adv Manuf Technol 40 (3-4):316-323

8. Zhang QH, Du R, Zhang JH, Zhang QB (2006) An investigation of ultrasonic-assisted electrical discharge machining in gas. Int J Mach Tools Manuf 46 (12-13):1582-1588

9. Tan PC, Yeo SH (2008) Modelling of overlapping craters in micro-electrical discharge machining. J Phys D-Appl Phys 41 (20)

10. Kurnia W, Tan PC, Yeo SH, Tan QP (2009) Surface roughness model for micro electrical discharge machining. P I Mech Eng B-J Eng 223 (3):279-287

11. Wang Y, Chen S-Y, Xiong W, Wu C-Z (2020) Study on workpiece surface forming mechanism by successive discharges during USV-MF complex-assisted WEDM-LS process. Int J Adv Manuf Technol 108 (9-10):2985-3000

12. Zhao Y, Zhang X, Liu X, Yamazaki K (2004) Geometric modeling of the linear motor driven electrical discharge machining (EDM) die-sinking process. Int J Mach Tools Manuf 44 (1):1-9

13. Izquierdo B, Sánchez JA, Plaza S, Pombo I, Ortega N (2009) A numerical model of the EDM process considering the effect of multiple discharges. Int J Mach Tools Manuf 49 (3-4):220-229

14. Liu JF, Guo YB (2016) Thermal Modeling of EDM with Progression of Massive Random Electrical Discharges. 
Procedia Manuf 5:495-507

15. Jithin S, Bhandarkar UV, Joshi SS (2017) Analytical Simulation of Random Textures Generated in Electrical Discharge Texturing. J Manuf Sci Eng 139 (11)

16. Jithin S, Raut A, Bhandarkar UV, Joshi SS (2020) Finite element model for topography prediction of electrical discharge textured surfaces considering multi-discharge phenomenon. Int J Mech Sci 177

17. Li Z, Bai J (2016) Impulse discharge method to investigate the influence of gap width on discharge characteristics in micro-EDM. Int J Adv Manuf Technol 90 (5-8):1769-1777

18. Li Z, Bai J, Zhu X (2016) Research on the Depth Error in Micro Electrical Discharge Milling. Procedia CIRP 42:638-643

19. Shabgard M, Ahmadi R, Seyedzavvar M, Oliaei SNB (2013) Mathematical and numerical modeling of the effect of input-parameters on the flushing efficiency of plasma channel in EDM process. Int J Mach Tools Manuf 65:79-87

20. Perveen A, Jahan MP (2018) Modeling and optimization of crater size generated during micro- EDM of Ti alloy using Response surface method. Mater Today P 5 (9):18307-18314

21. Paul G, Roy S, Sarkar S, Hanumaiah N, Mitra S (2012) Investigations on influence of process variables on crater dimensions in micro-EDM of $\gamma$-titanium aluminide alloy in dry and oil dielectric media. Int J Adv Manuf Technol 65 (5-8):1009-1017

22. Mascaraque-Ramírez C, Franco P (2019) Comparison between different methods for experimental analysis of surface integrity in die-sinking electro-discharge machining processes. P I Mech Eng B-J Eng 234 (3):479-488

23. Liu Q, Zhang Q, Zhang M, Yang F (2020) Study on the Discharge Characteristics of Single-Pulse Discharge in Micro-EDM. Micromachines 11 (1)

24. Liu Q, Zhang Q, Zhang M, Zhang J (2016) Review of size effects in micro electrical discharge machining. Precis Eng 44:29-40

25. Wong YS, Rahman M, Lim HS, Han H, Ravi N (2003) Investigation of micro-EDM material removal characteristics using single RC-pulse discharges. J Mater Process Technol 140 (1-3):303-307

26. Li Z, Bai J, Tang J (2018) Experimental investigation on the discharge stability in micro-EDM based on finite-successive pulses discharge method. J Phys D-Appl Phys 51 (47)

27. Rohatgi VK, Saleh AME (2001) An introduction to probability and statistics. Second edn. Wiley Interscience, New York

28. Tricarico C, Delpretti R, Dauw DF (1988) Geometrical Simulation of the EDM Die-Sinking Process. Ann CIRP 37 (1):191-196

29. Kojima A, Natsu W, Kunieda M (2008) Spectroscopic measurement of arc plasma diameter in EDM. Ann CIRP 57 (1):203-207

30. Zhang L, Du J, Zhuang X, Wang Z, Pei J (2015) Geometric prediction of conic tool in micro-EDM milling with fix-length compensation using simulation. Int J Mach Tools Manuf 89:86-94

31. Jeong YH, Min B-K (2007) Geometry prediction of EDM-drilled holes and tool electrode shapes of micro-EDM process using simulation. Int J Mach Tools Manuf 47 (12-13):1817-1826

32. Antar M, Hayward P, Dunleavey J, Butler-Smith P (2018) Surface Integrity Evaluation of Modified EDM Surface Structure. Procedia CIRP 68:308-312 


\section{Figures}
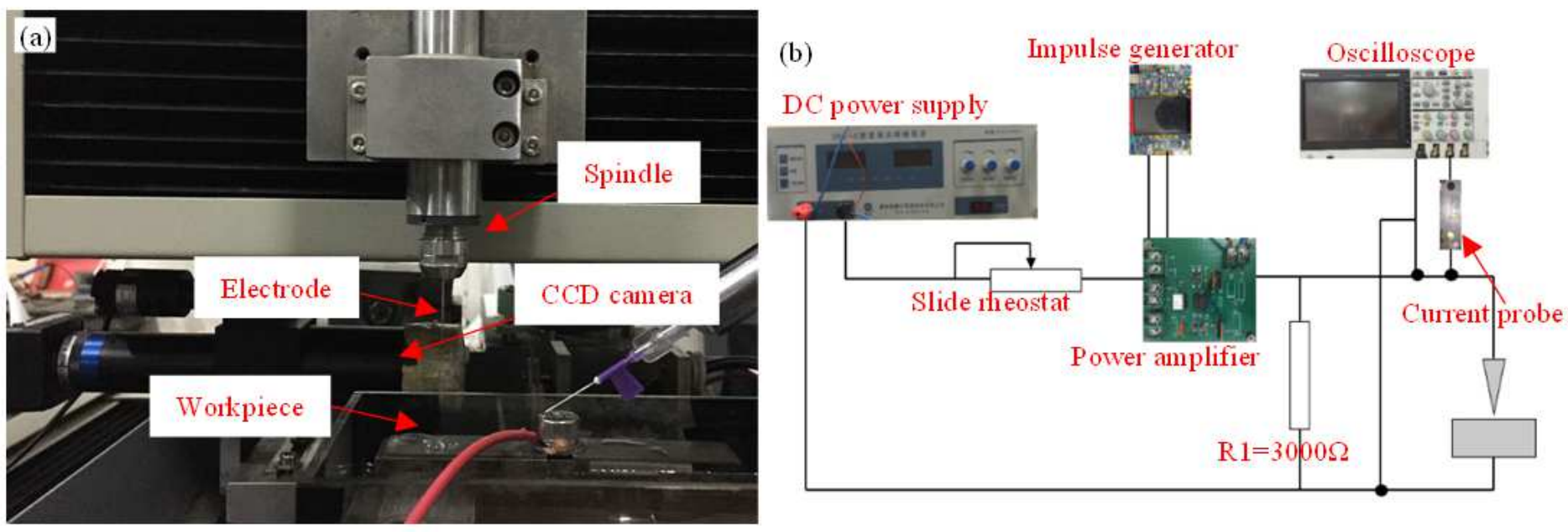

Figure 1

(a) machine tool and (b) discharge circuit.

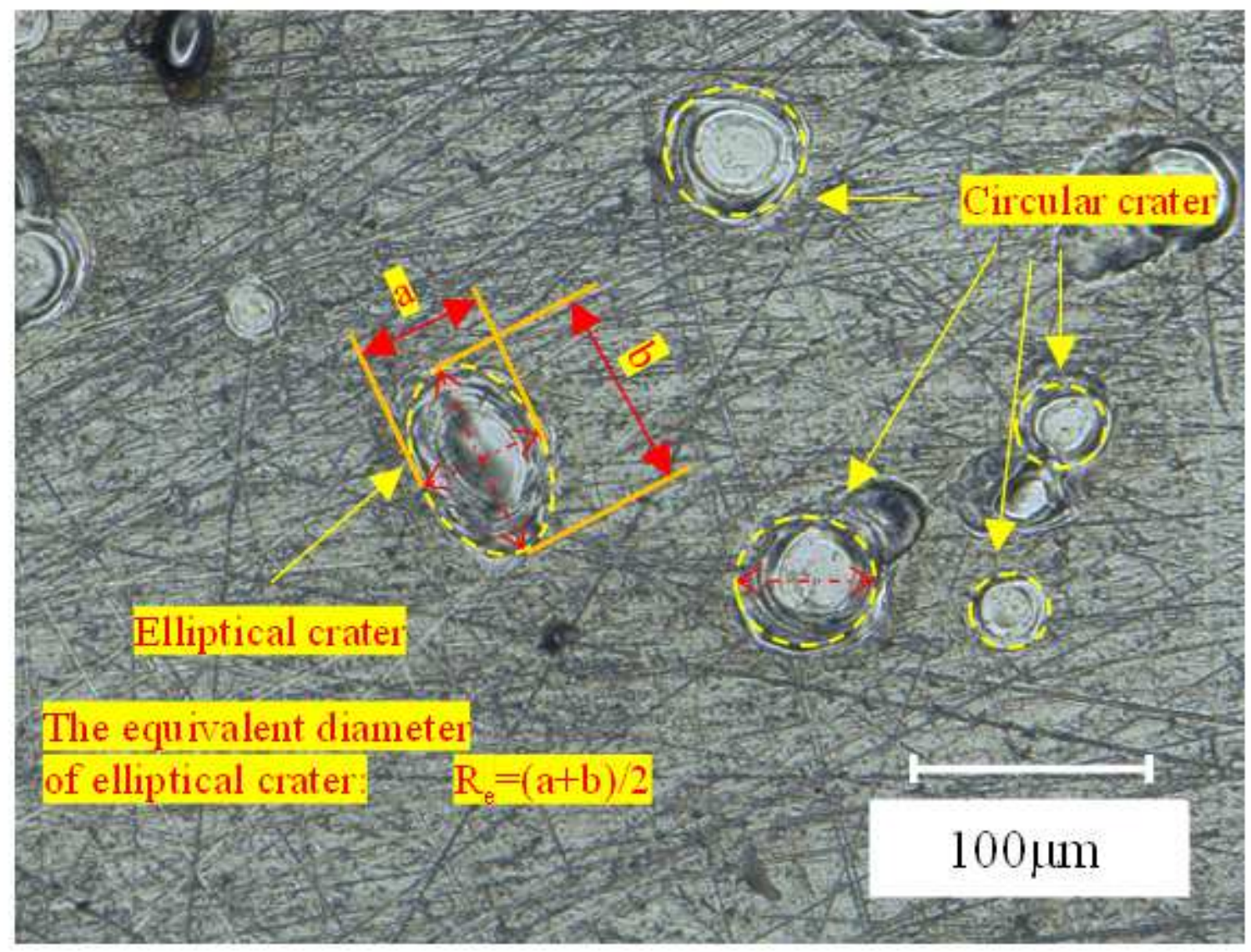

\section{Figure 2}

The two types of discharge crater 

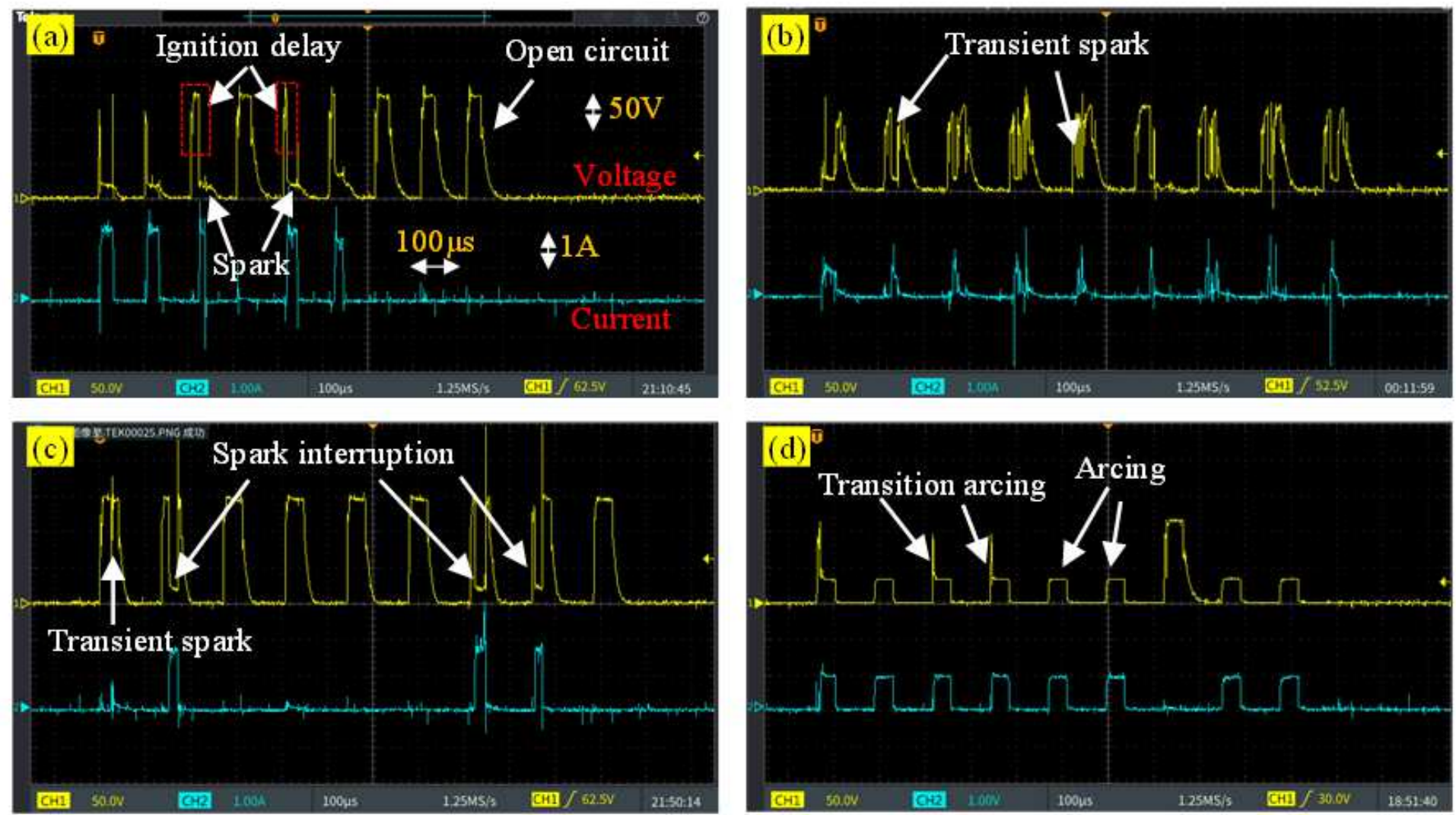

\section{Figure 3}

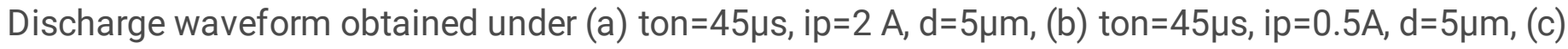

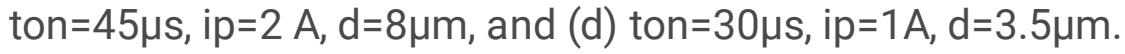

(a)

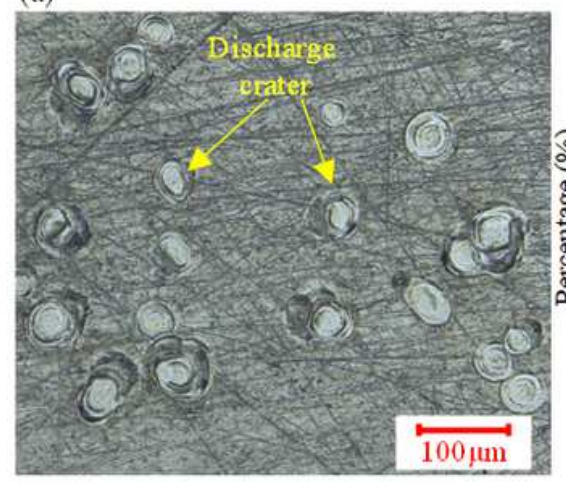

(b)

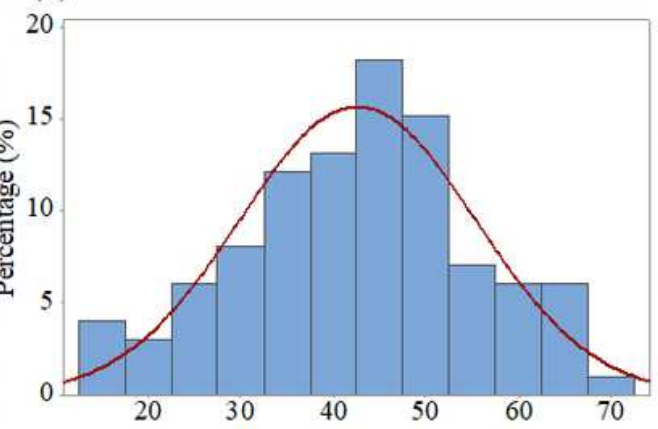

Diameter of discharge craters in Exp.2 ( $\mu \mathrm{m})$ (c)

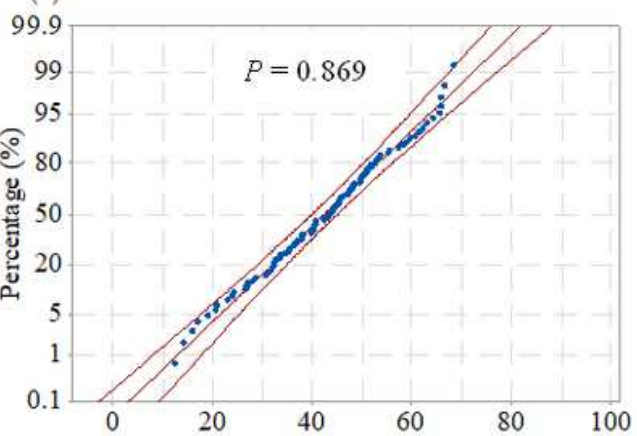

Diameter of discharge craters in Exp.2 $(\mu \mathrm{m})$

\section{Figure 4}

(a) Distribution of discharge craters of exp. 2, (b) histogram of crater diameter distribution and (c) goodness-of-fit test for normal distribution. 


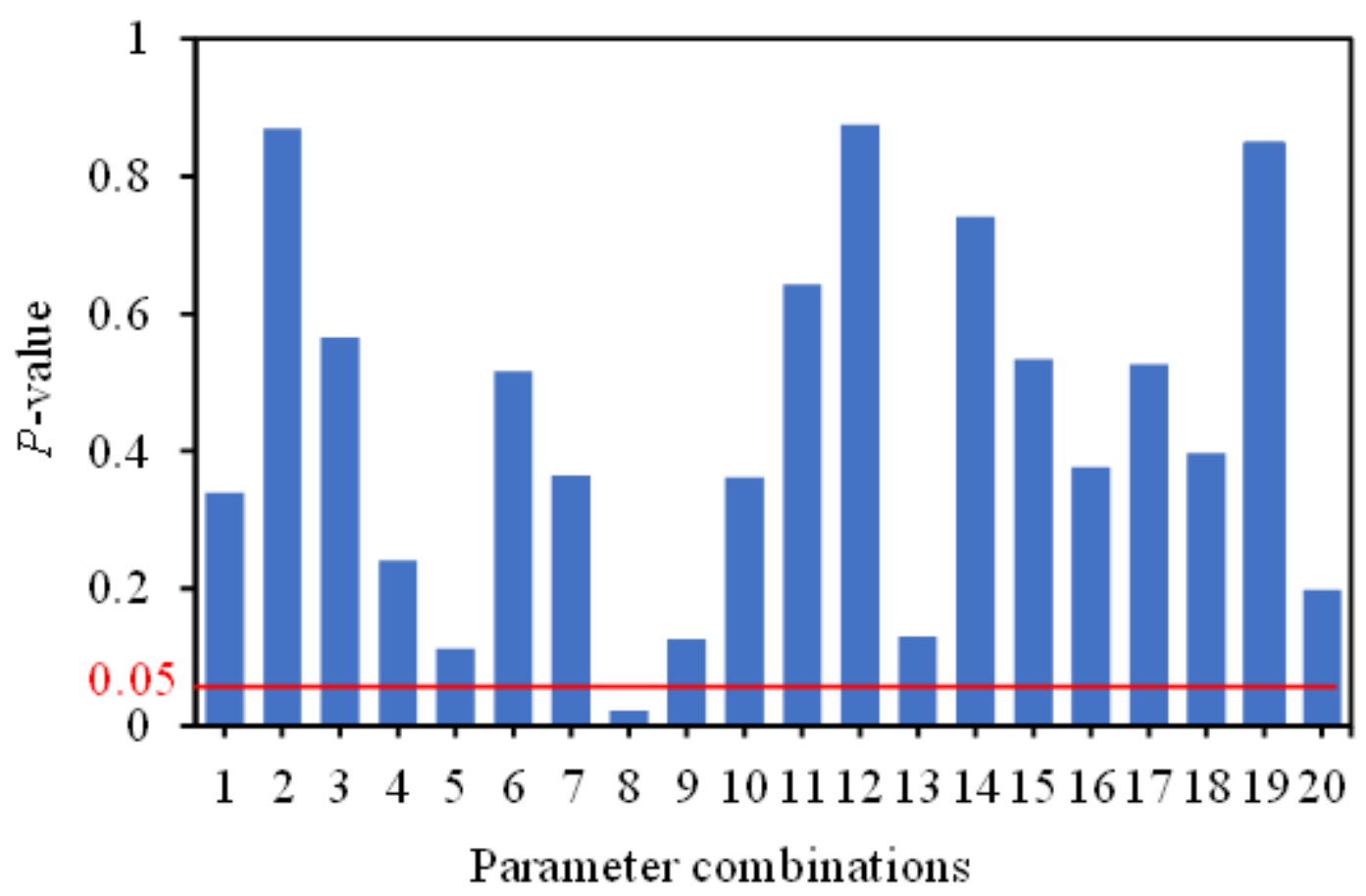

Figure 5

The P-value of goodness-of-fit test for normal distribution of the 20 groups of data.
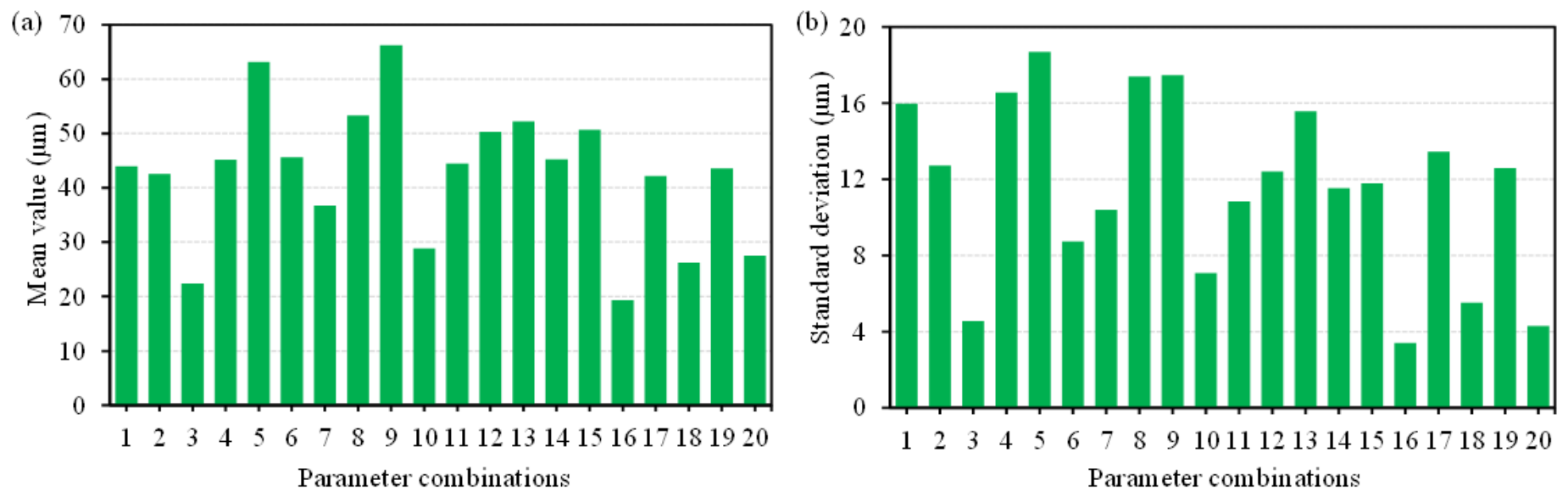

Figure 6

Mean value and standard deviation of crater size under different parameter combinations. 


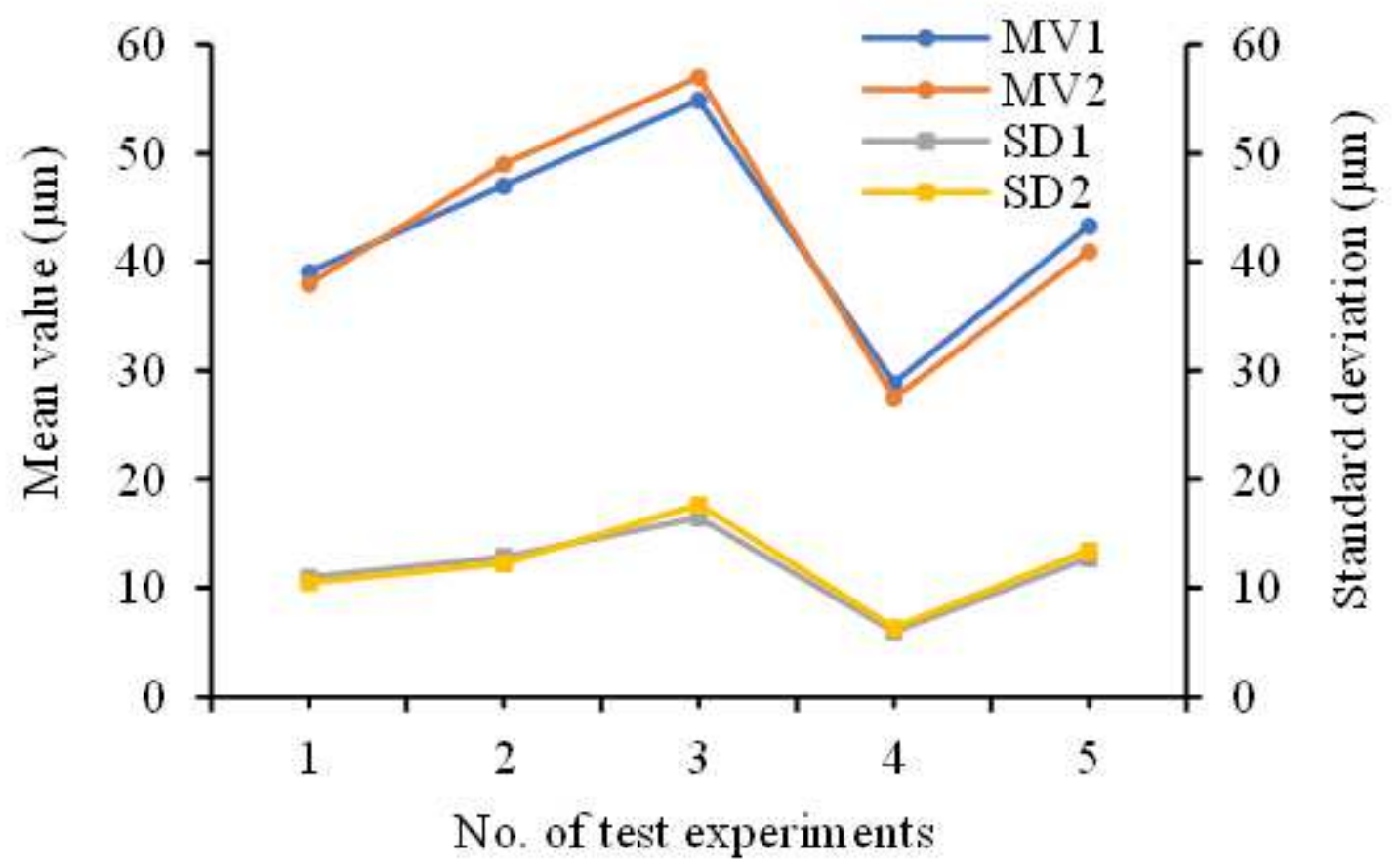

Figure 7

Experimental and predictive results for crater size

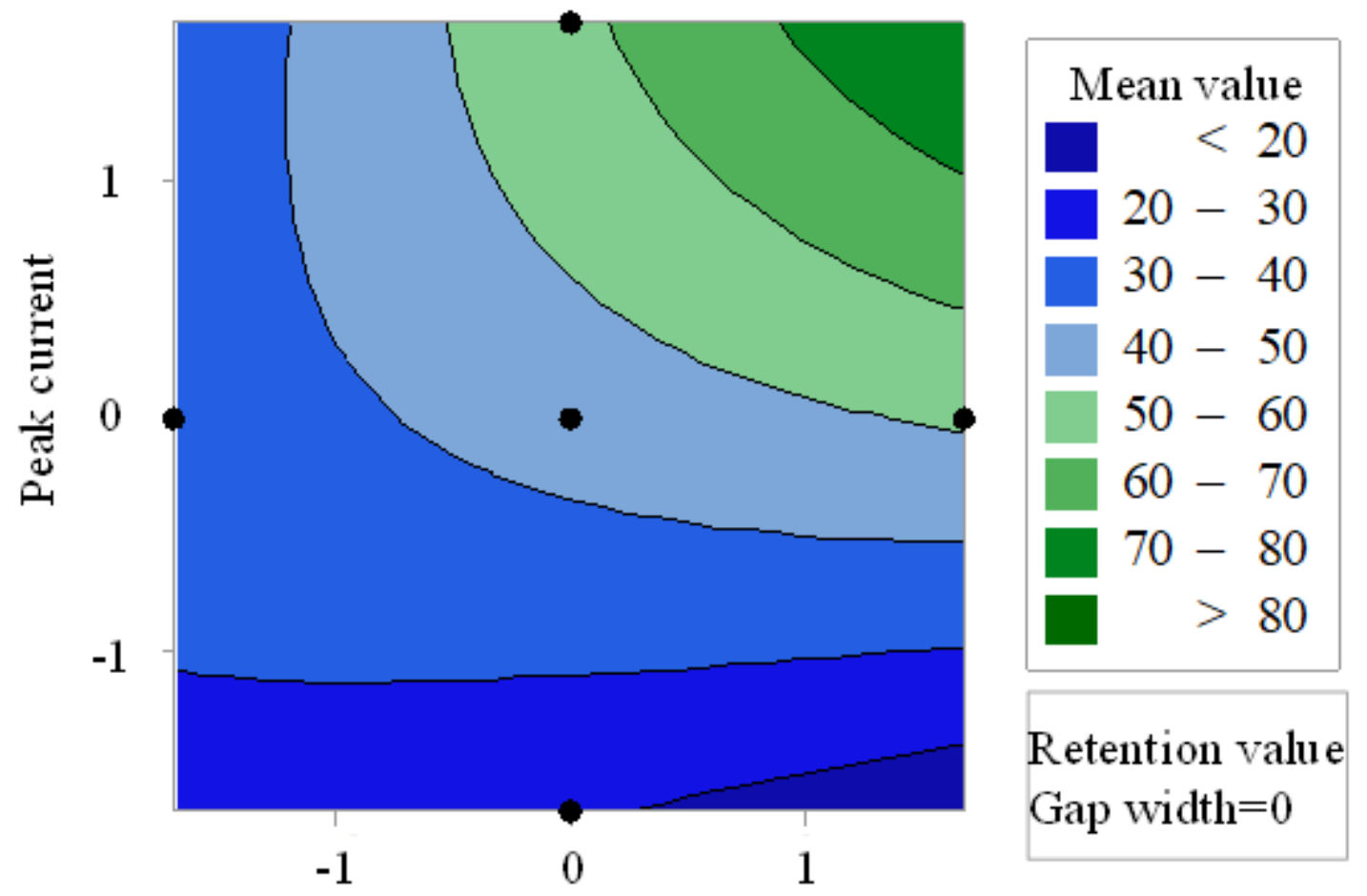

Pulse width

Figure 8 
Contour plot for mean value vs ip and $d$

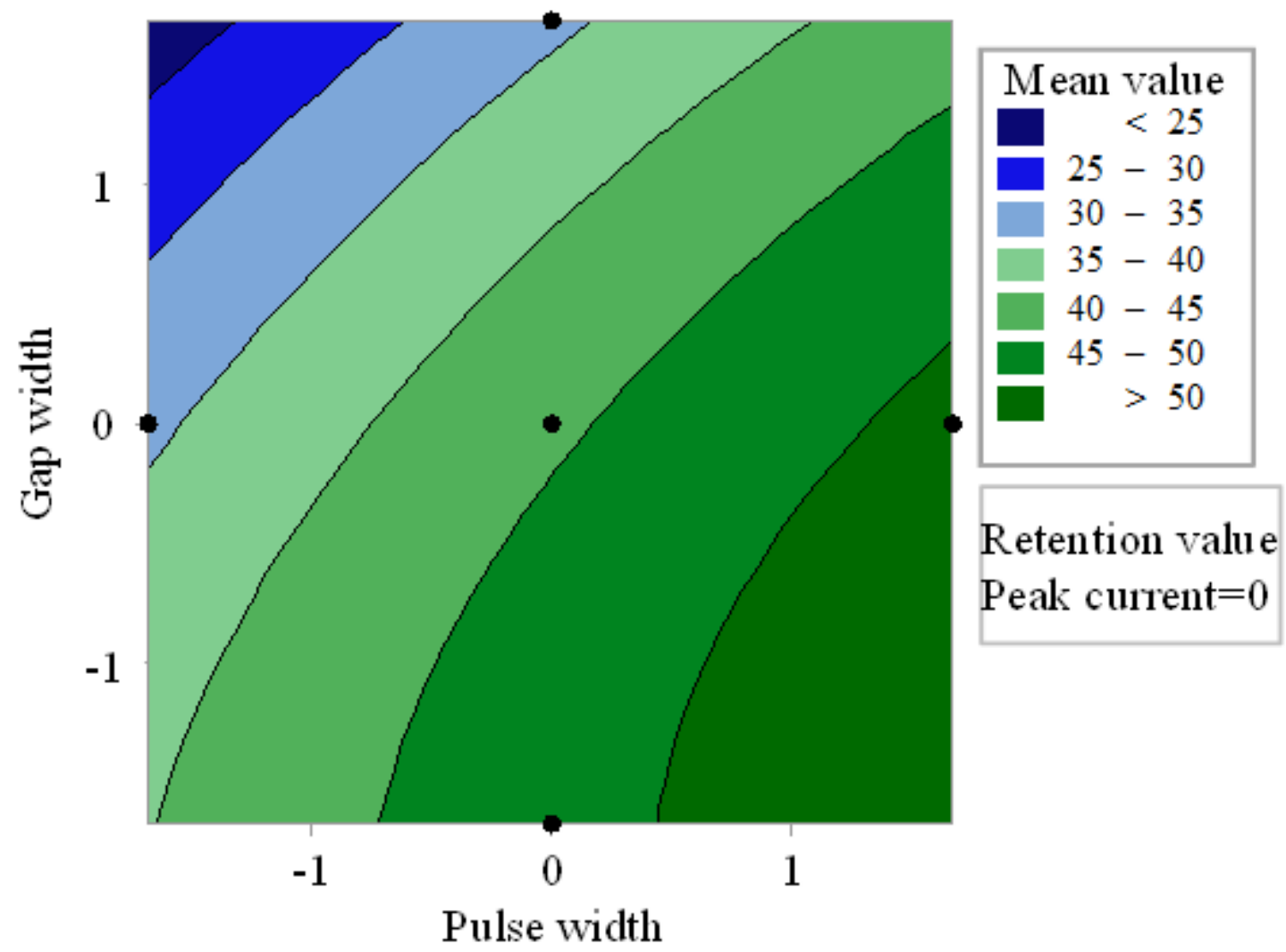

Figure 9

Contour plot for mean value vs $\mathrm{d}$ and $\mathrm{t}$ 


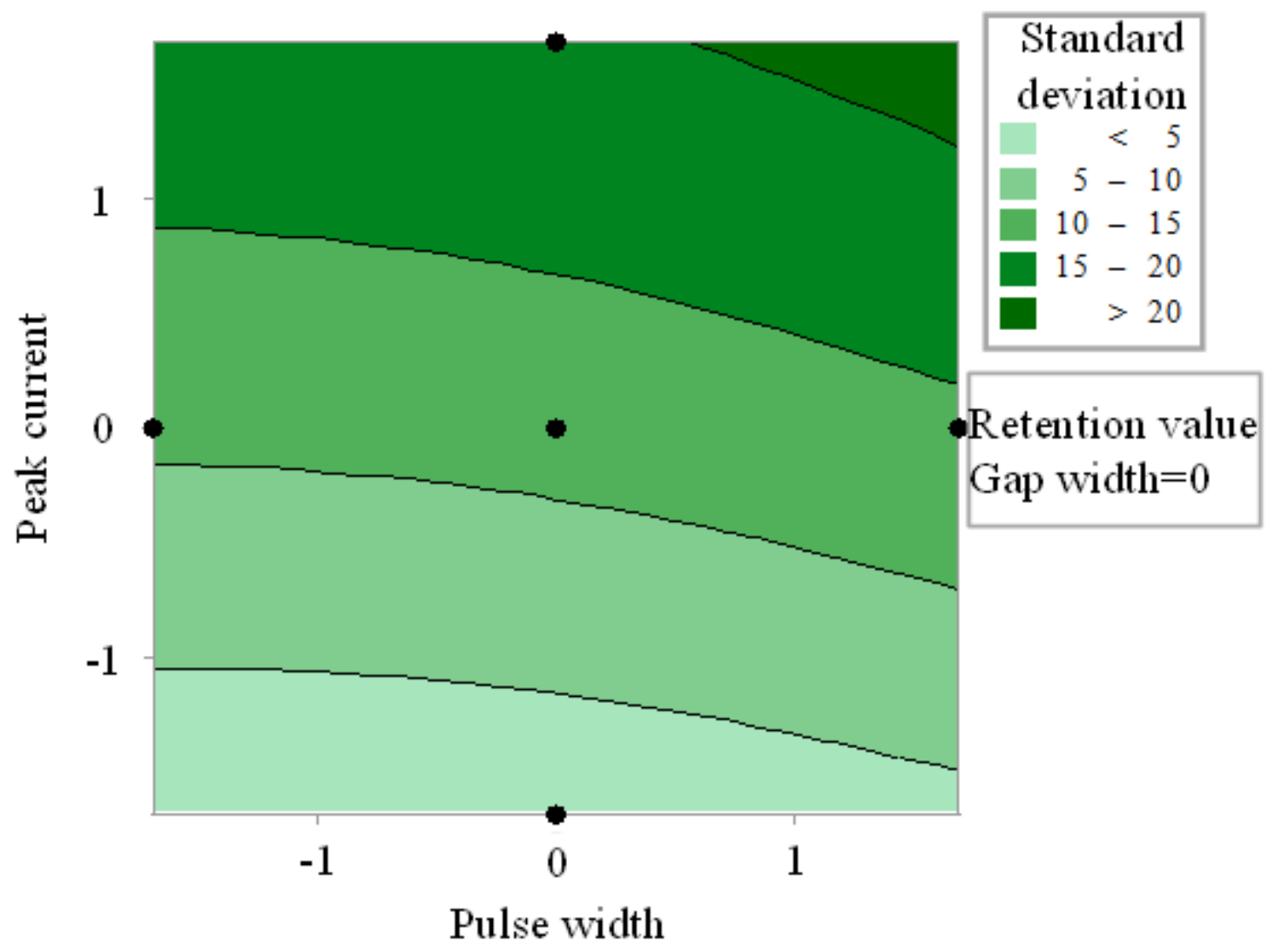

Figure 10

Contour plot for standard deviation vs ip and tp 


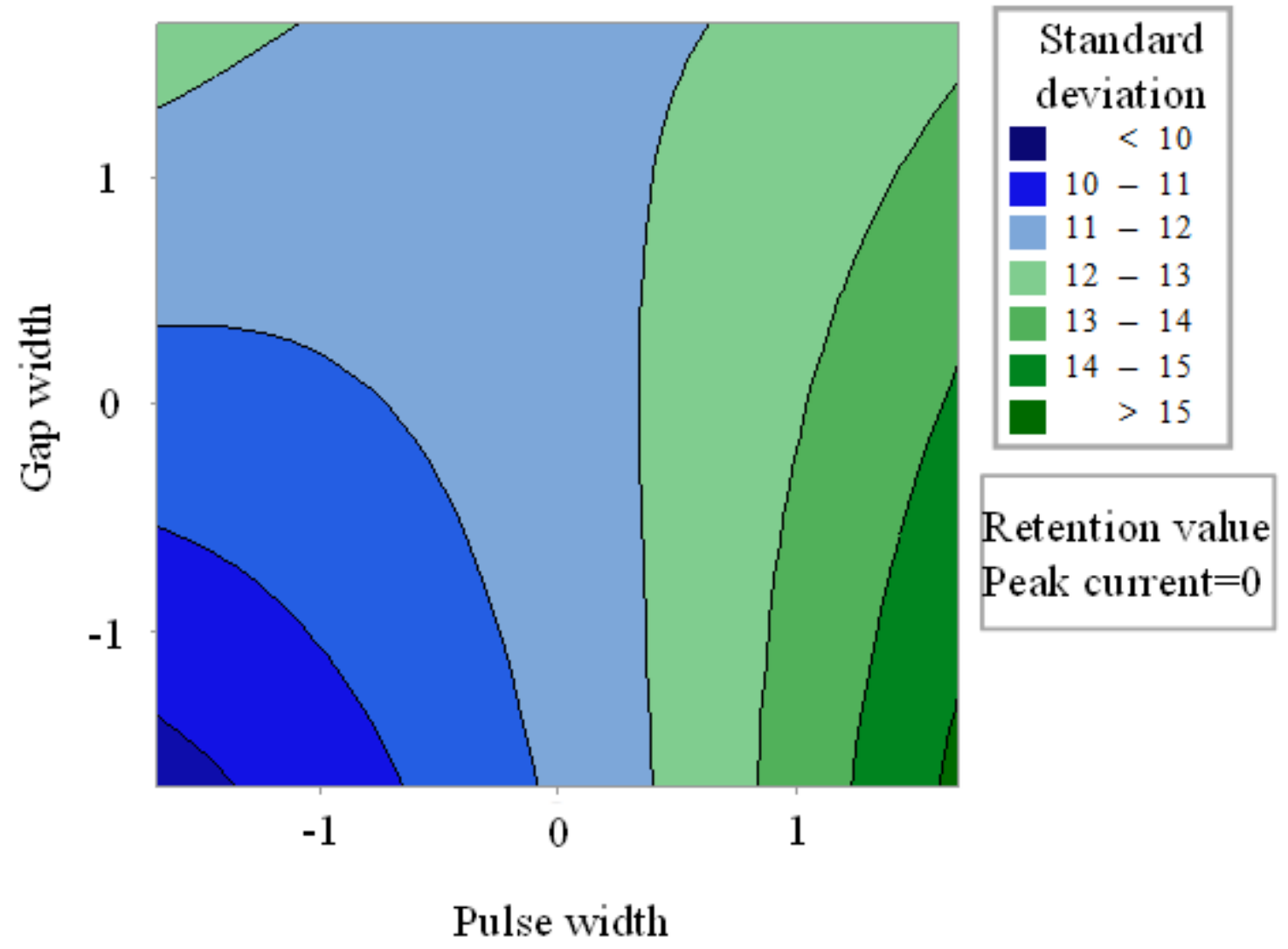

Figure 11

Contour plot for standard deviation vs $d$ and tp

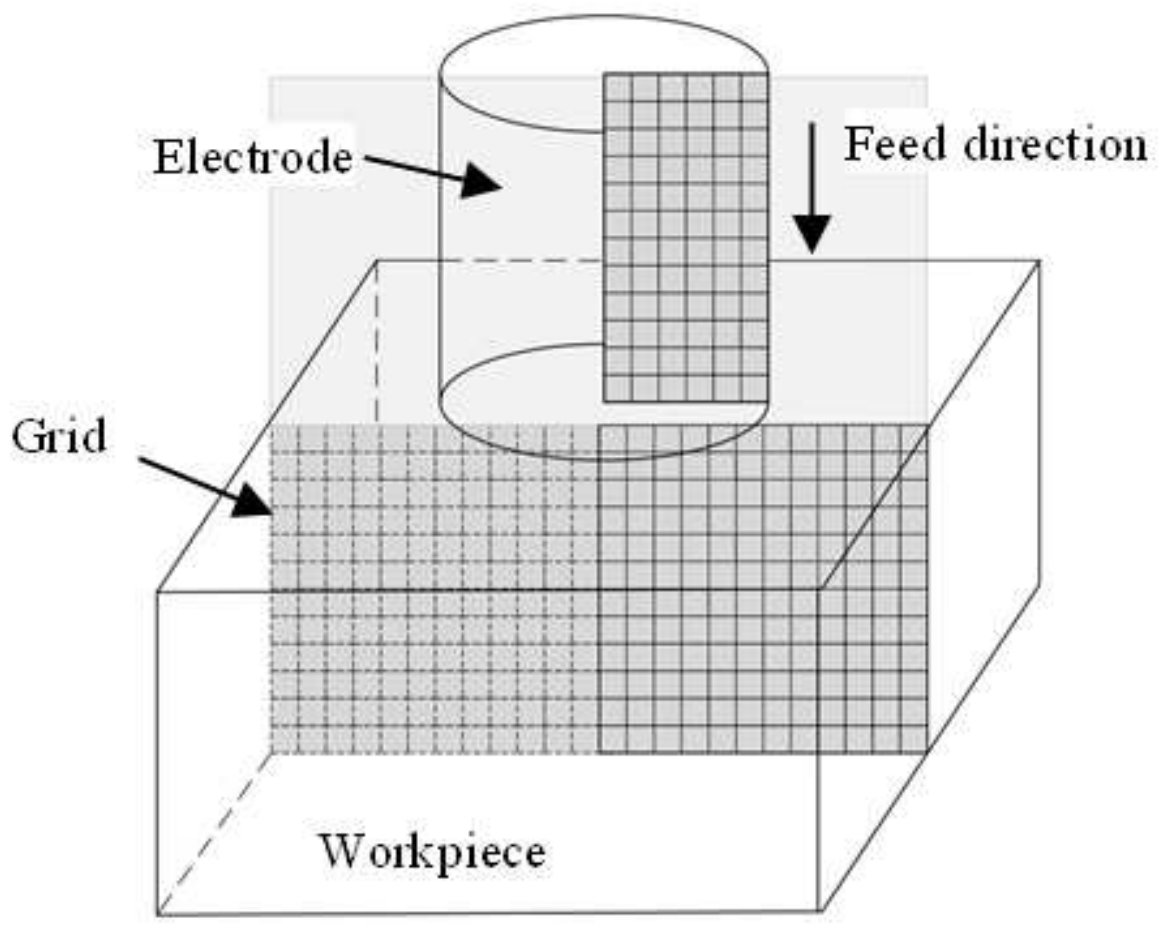

Figure 12 


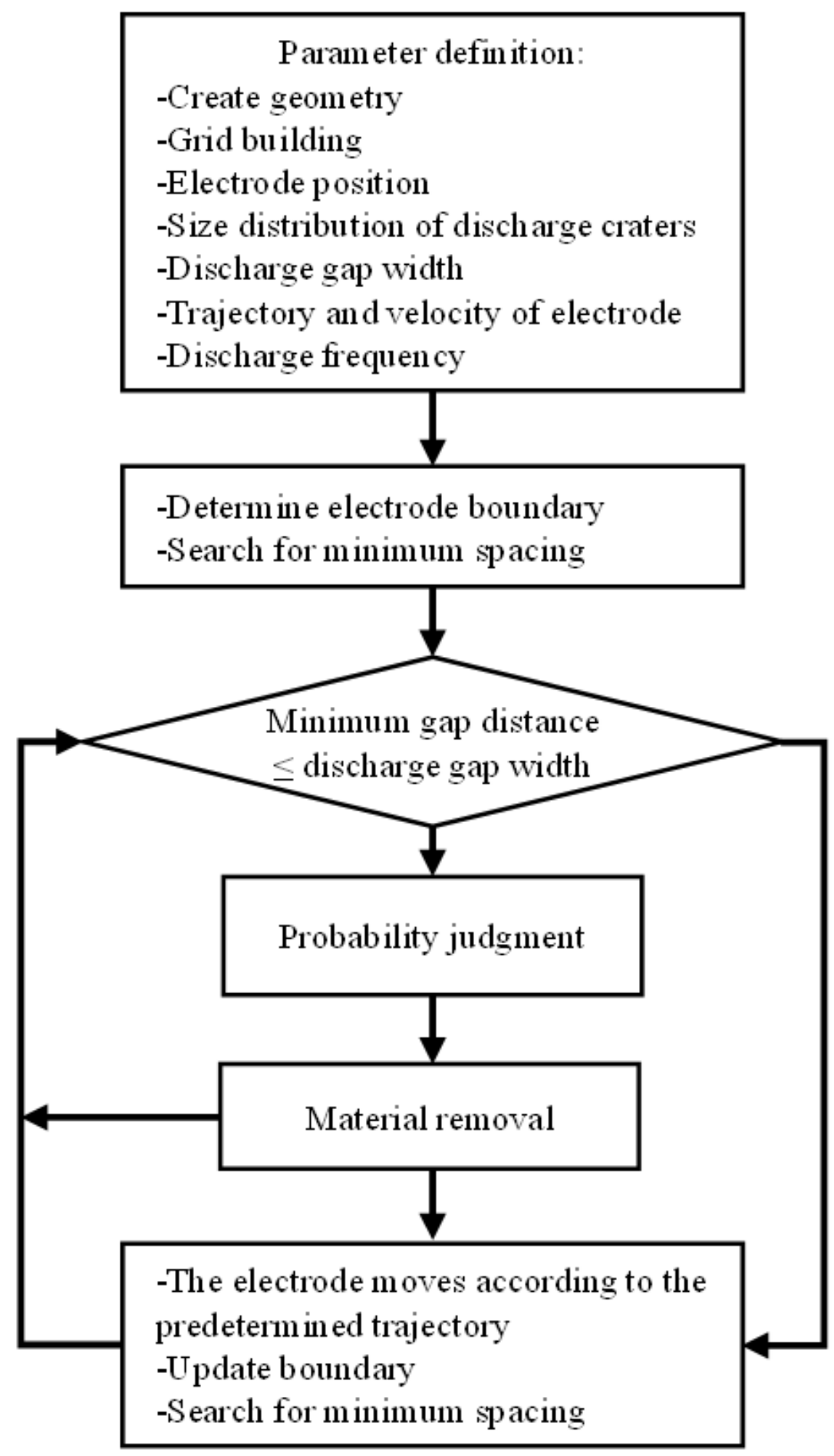

Figure 13

Flow chart of the simulation model 

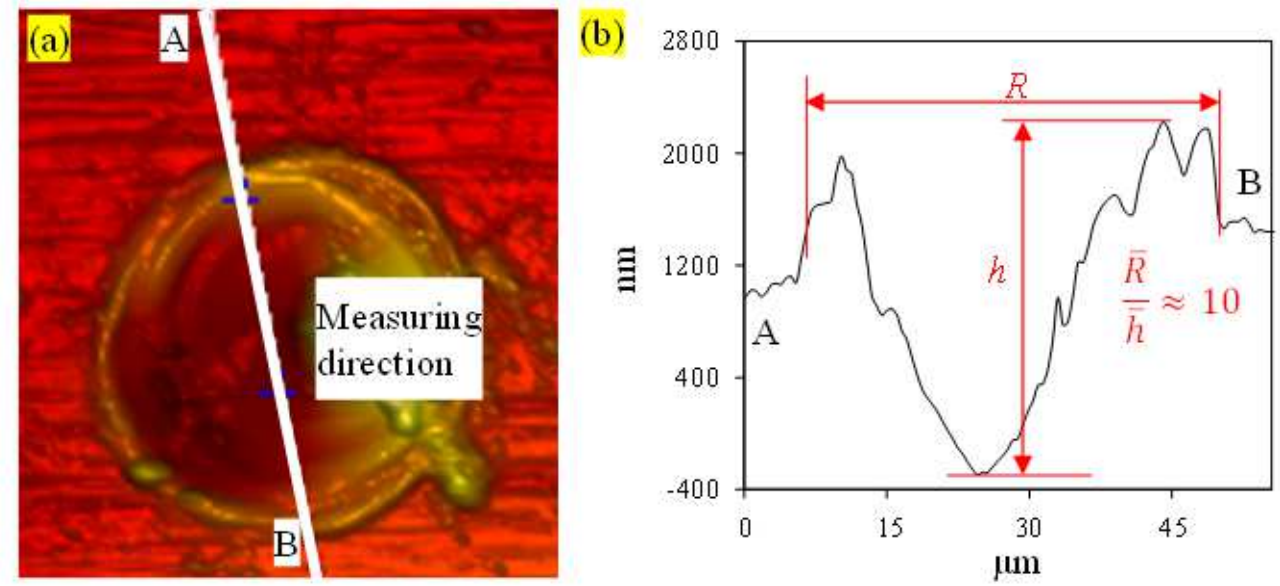

(c)

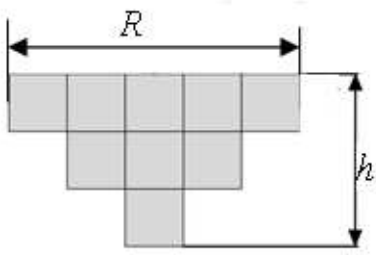

Figure 14

The morphology of discharge crater: (a) AFM photo, (b) cross section and (c) Equivalent geometric model of crater.

The mean value and standard deviation of the chameter of the discharge pit.

Determine the normal probability function $y=f(x)$

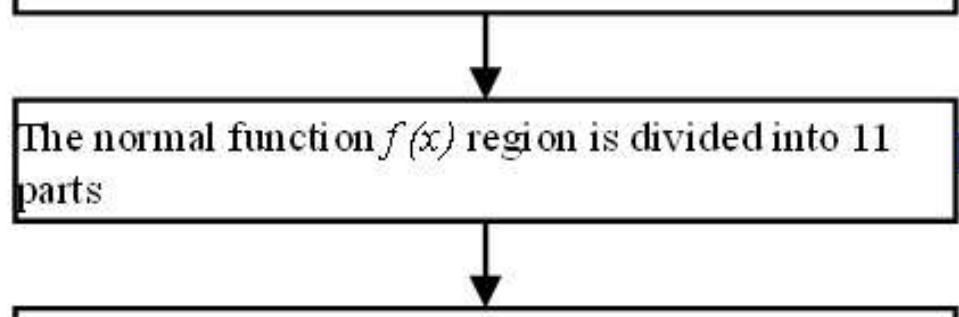

The interval $[0,1]$ is divided into 11 intervals:

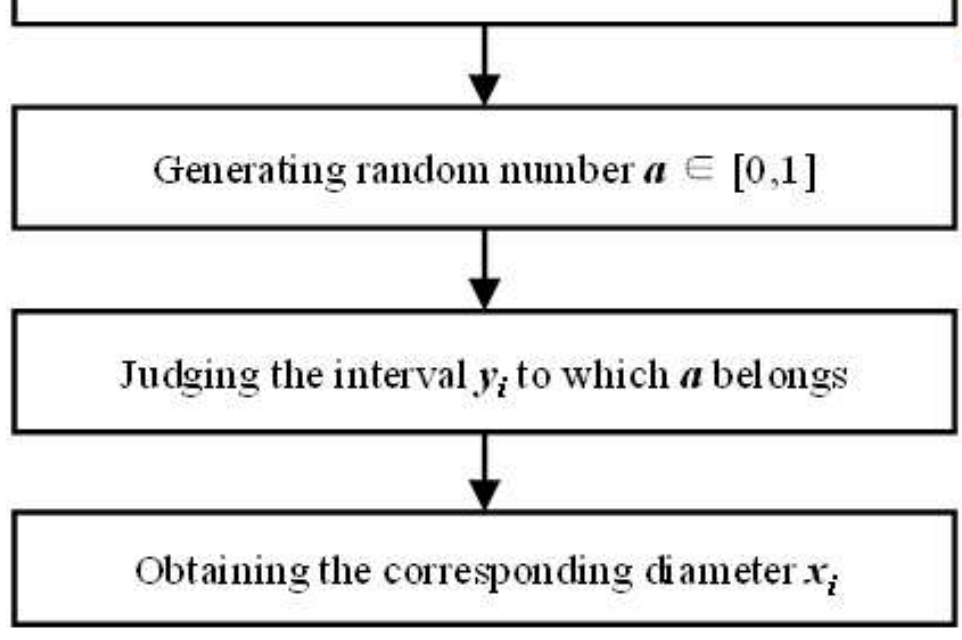

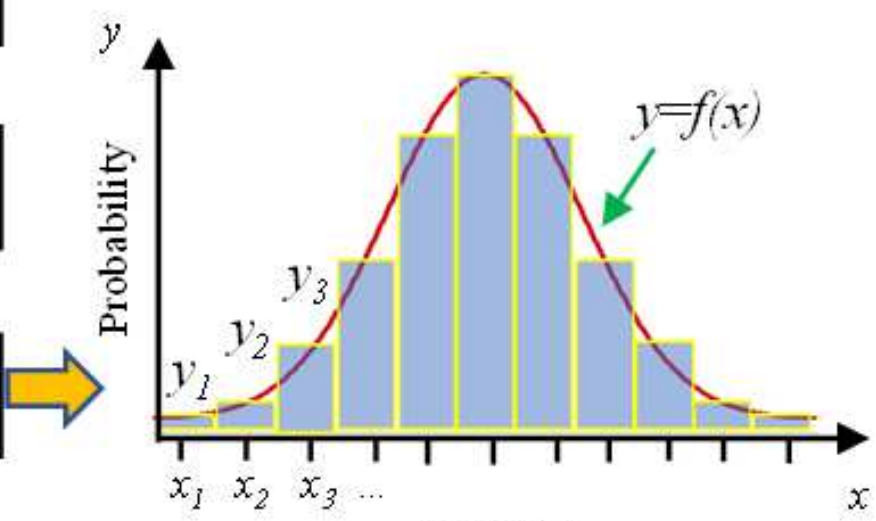

Diameter

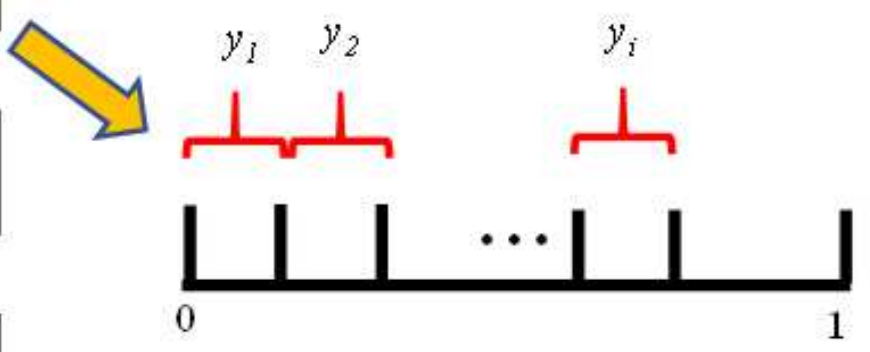

$$
y_{1}+y_{2}+\ldots+y_{i}+\ldots y_{11}=1
$$


Flow chart of judging discharge crater size
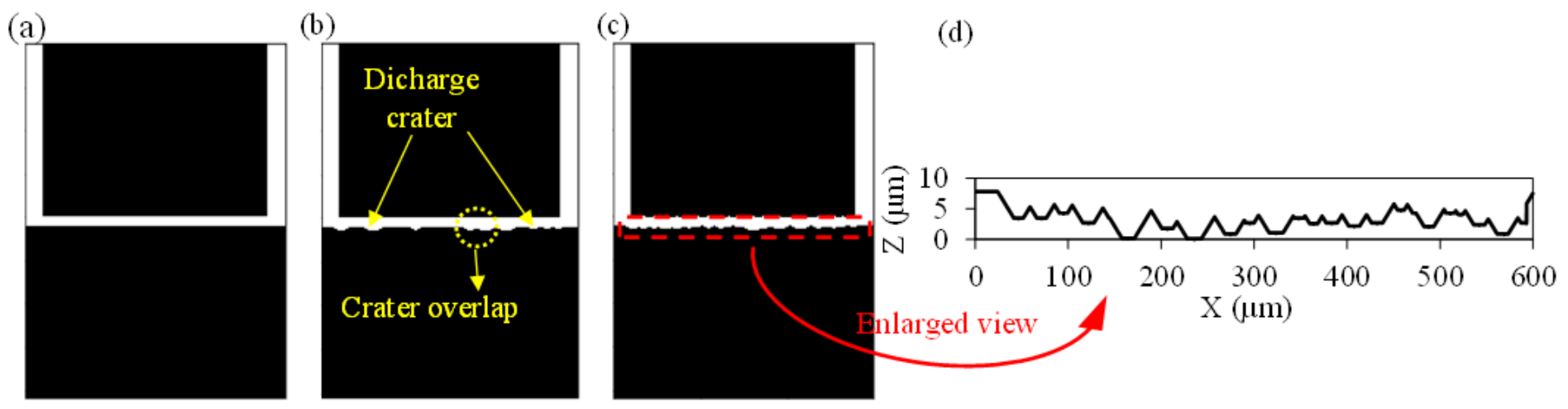

Figure 16

Evolution of surface morphology of MSDS

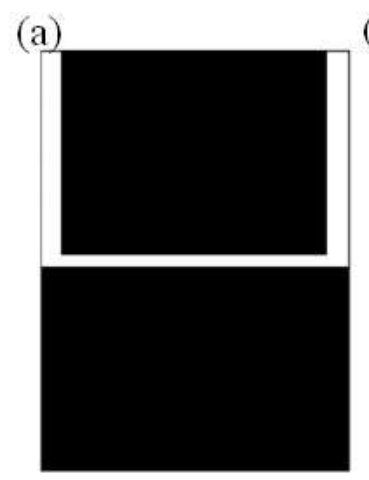

(b)

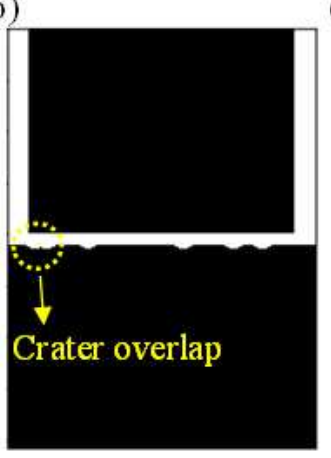

(c)

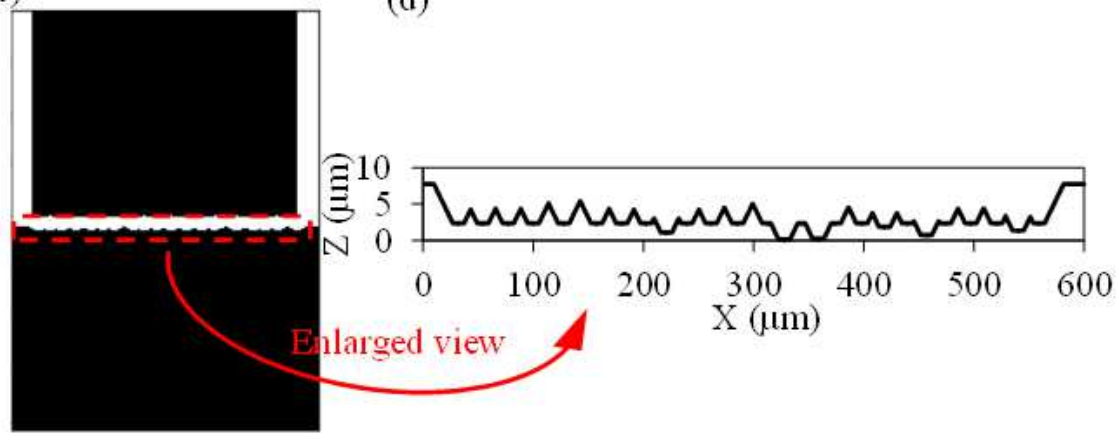

Figure 17

Evolution of surface morphology of MFS

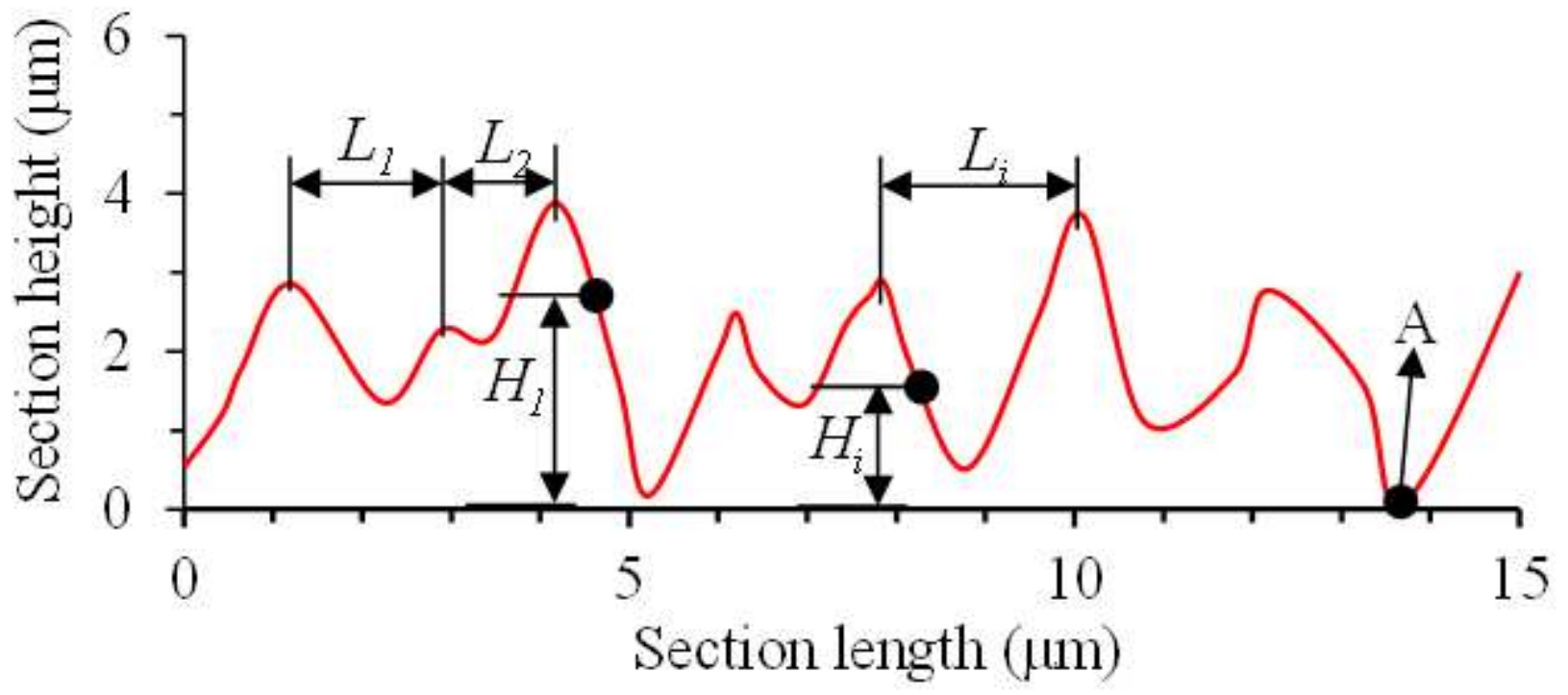


Figure 18

The schematic diagram of cross section morphology

(a)

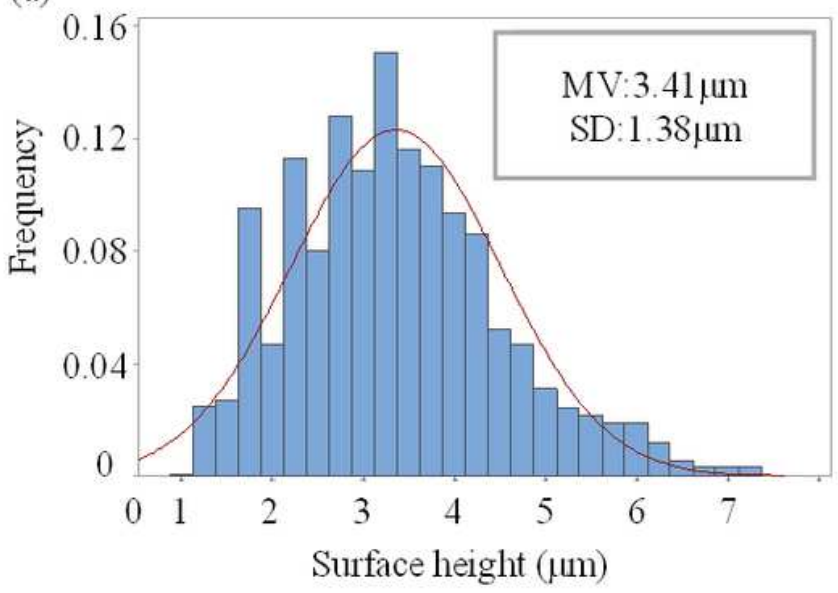

(c)

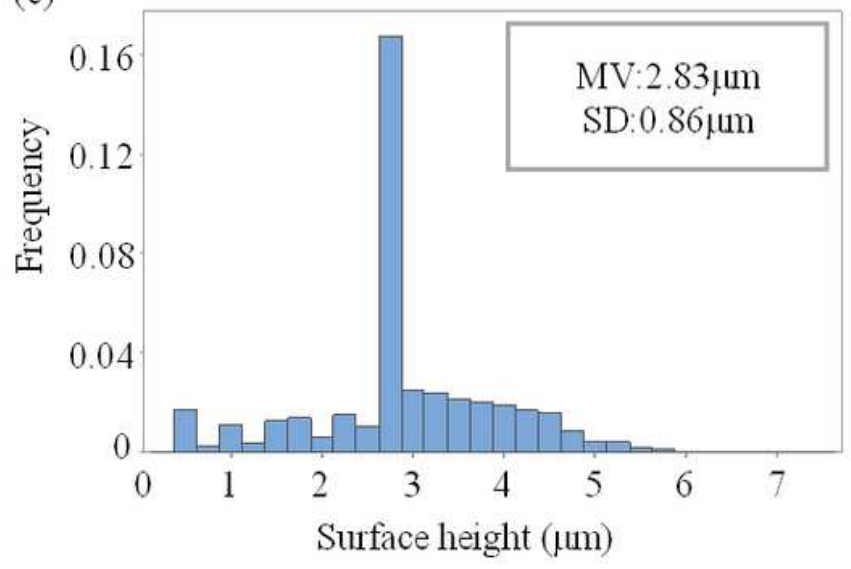

(b)

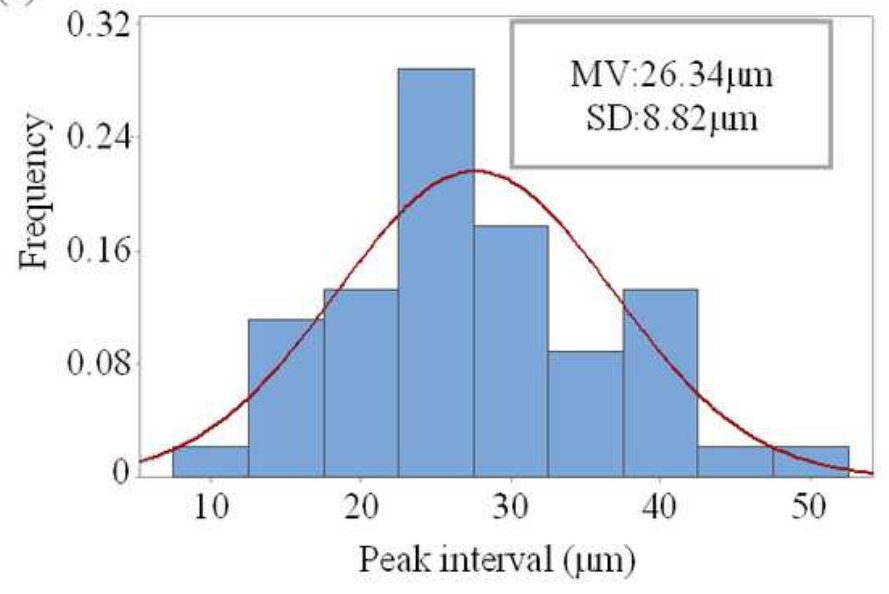

(d)

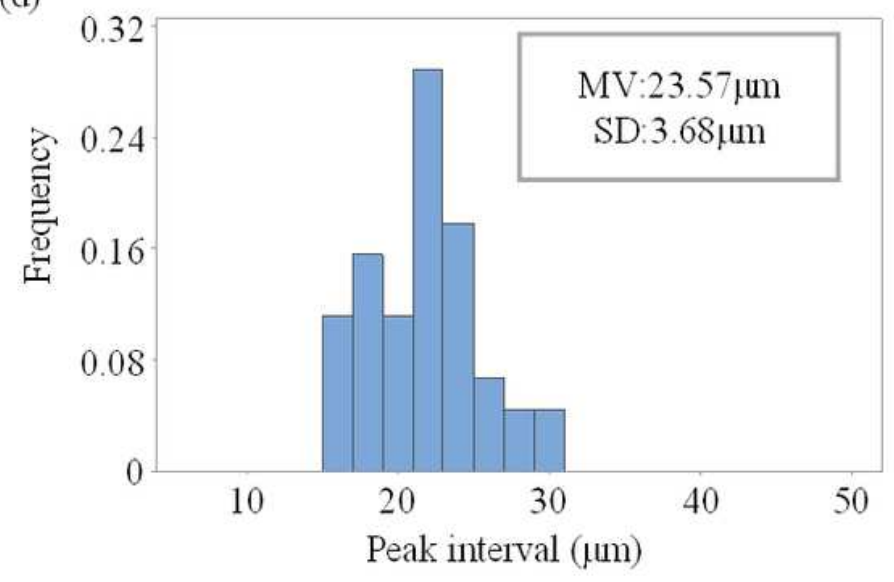

Figure 19

The distribution characteristics of (a) (c) the surface height and (b) (d) interval of adjacent peaks for MSDS and MFS separately. 
(a)

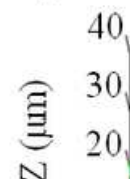

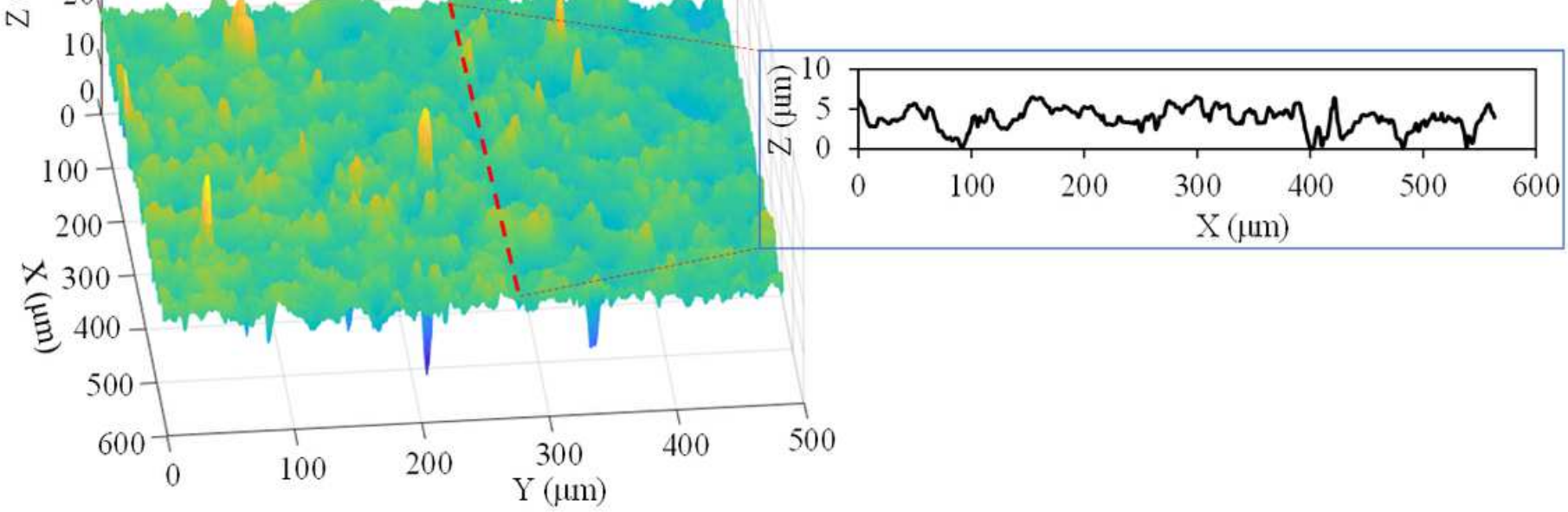

(b)

\section{Figure 20}

The (a) 3D Morphology and (b) 2D Morphology of the machined surface.

(a)

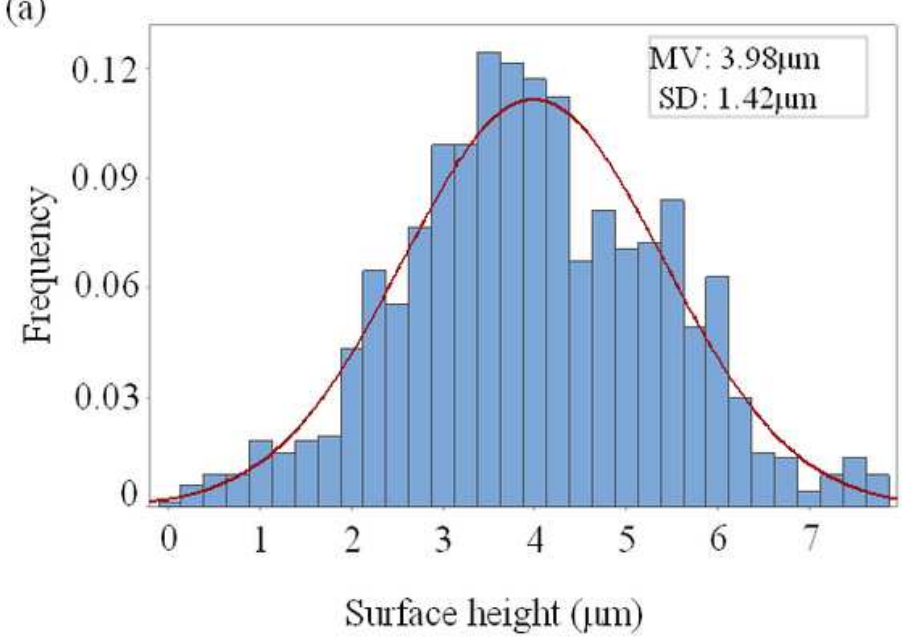

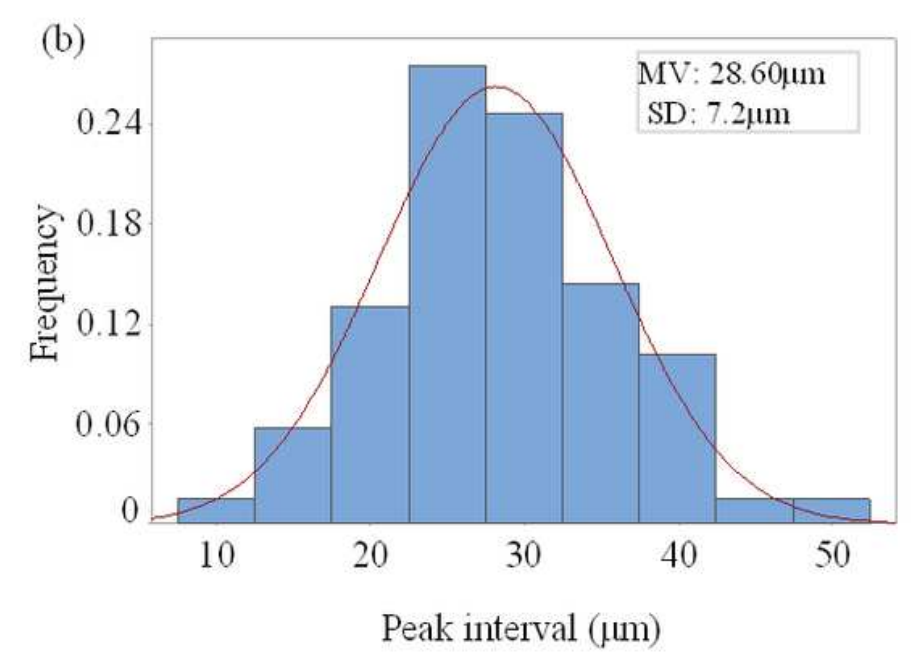

\section{Figure 21}

The distribution characteristics of the surface height and interval of adjacent peaks for machined surface 

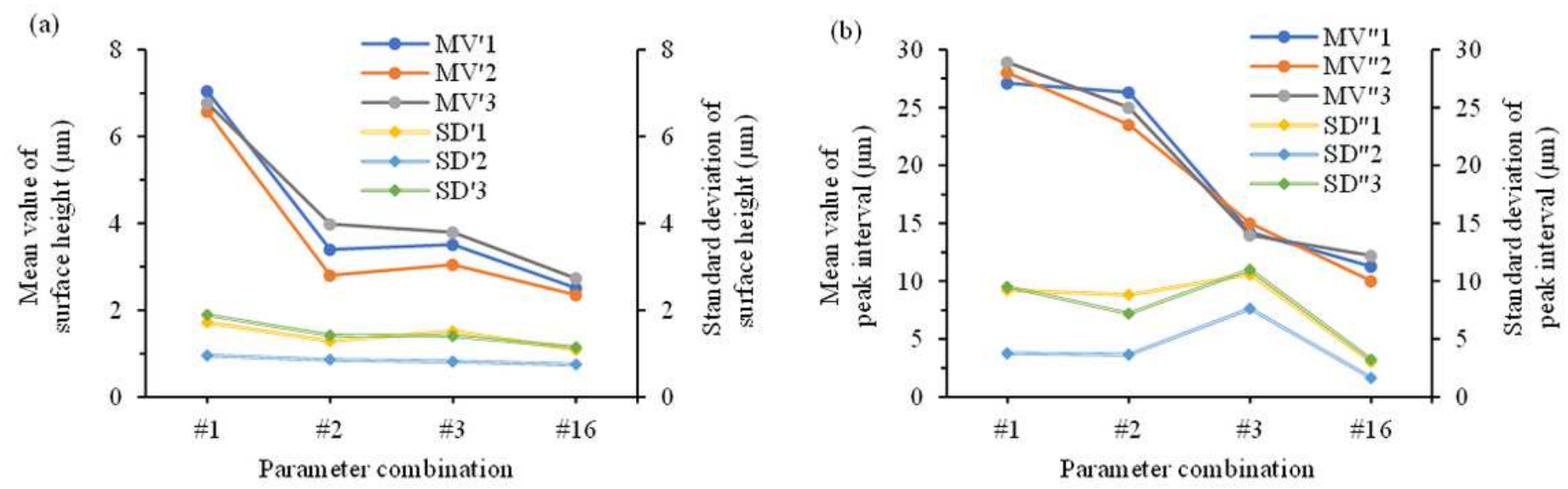

Figure 22

Comparison for (a) surface height and (b) peak interval of the simulated surface and the machined surface. 